\title{
Masonry structures in the presence of foundation settlements and unilateral contact problems
}

Antonio Tralli ${ }^{\text {a }}$, Andrea Chiozzi ${ }^{\text {a* }}$, Nicola Grillanda ${ }^{\text {b }}$, Gabriele Milani ${ }^{\text {b }}$

a Department of Engineering, University of Ferrara, Via Saragat 1, 44122 - Ferrara (Italy)

${ }^{b}$ Department of Architecture, Built Environment and Construction Engineering (A.B.C.), Technical University of Milan,

Via Ponzio 31, 20133 - Milan (Italy)

\begin{abstract}
This paper aims at presenting a new perspective on the modeling of the influence of foundation settlements in masonry structures. We show that it is possible to model in a consistent way the crack pattern and the associated mechanism induced by applied settlements starting from a proper treatment of unilateral contact constraints between contiguous blocks, in which the structure is partitioned after the onset of settlements. We extend two classic variational formulations for the Signorini problem, namely the minimum of the total potential energy and a complementary boundary formulation, to the case of contact between multiple no-tension rigid bodies satisfying Heyman's hypotheses for the limit analysis of masonry structures. The discretization of the proposed variational formulations directly entails two dual linear programming problems. The proposed formulations allow to correctly evaluate the effects of foundation settlements and their influence on the ultimate load bearing capacity of masonry structures.
\end{abstract}

Keywords: masonry structures; foundation settlements; rigid no-tension material; unilateral contact; limit analysis

* Corresponding Author

Email: andrea.chiozzi@unife.it

Phone: +393474027959 


\section{Introduction}

Masonry constructions represent an important part of both Eastern and Western built environment and certainly the prevailing one among historical constructions making up world architectural heritage. The study of historical masonry buildings from a structural standpoint deals with two main aspects: 1) understanding their structural behavior in terms of admissible states of equilibrium, to assess safety against applied loads, and 2) understanding the origin and significance of existing cracks, by establishing, for instance, what kind of foundation settlements gave origin to the observed crack pattern. With regard to the first aspect, it is widely acknowledged that modern theory of limit analysis of masonry structures, developed mainly by J. Heyman in the sixties (Heyman, 1969, 1966), is the most reliable tool to understand and analyze equilibrium of masonry structures, see, e.g., (Como, 2013; Huerta, 2011). A huge amount of works has covered this subject and, currently, the most efficient computational algorithms for the limit analysis of masonry structures are based on linear programming techniques (Boothby, 2001; Tralli et al., 2014); we also refer to (Galassi and Tempesta, 2019; Tempesta and Galassi, 2019) for the latest developments on Heyman's theory. Heyman introduced the following assumptions for analyzing the collapse of masonry structures:

i. Masonry is incapable of withstanding tension;

ii. Masonry has infinite compressive strength;

iii. Sliding cannot occur;

iv. Elastic strains are negligible.

According to these hypotheses, it is possible to prove that masonry material is stable according to the definition given by Drucker (Drucker, 1959) and rigorously state the two classical limit analysis theorems (Del Piero, 1998). Indeed, Heyman's model considers a masonry body as an assemblage of particles held together by the compressive stresses produced by loads. The small size of the stones, compared to the dimensions of the body, sometimes enables to consider the masonry structure as a continuum, instead of a discrete system of many individual particles. However, at the state of incipient collapse, cracks appear in masonry, actually identifying separate blocks of material. The displacement field is not continuous anymore and jumps appear at the interfaces between contiguous blocks: the structure, in fact, behaves like a mechanism (see Fig. 1a). On the other hand, more recent but growing is the interest in the study of the crack patterns induced by foundation settlements in masonry structures and the development of approximate numerical techniques to evaluate them, which often lead to the use of linear programming algorithms. Without the intention of being exhaustive, works by (Coccia et al., 2015; Dalgic et al., 2018; Galassi et al., 2013; Iannuzzo et al., 2018; Zampieri et al., 2017, 2018a) tackle the problem of foundation settlements in masonry structures, especially arch 
bridges, by treating masonry as a continuum, whereas the works by (Angelillo et al., 2018; Di Carlo et al., 2018; Galassi et al., 2018; Portioli and Cascini, 2017, 2016; Romano and Ochsendorf, 2010; Zampieri et al., 2019, 2018b) analyze masonry structures undergoing settlements as composed by a finite number of rigid blocks.

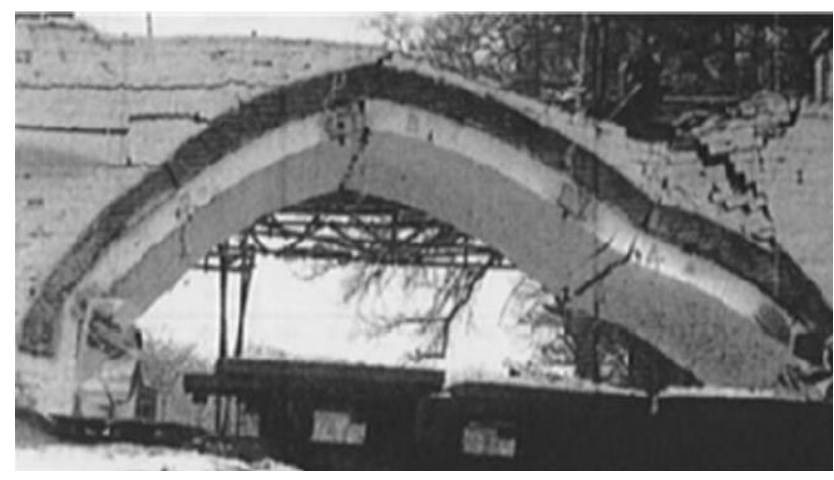

a

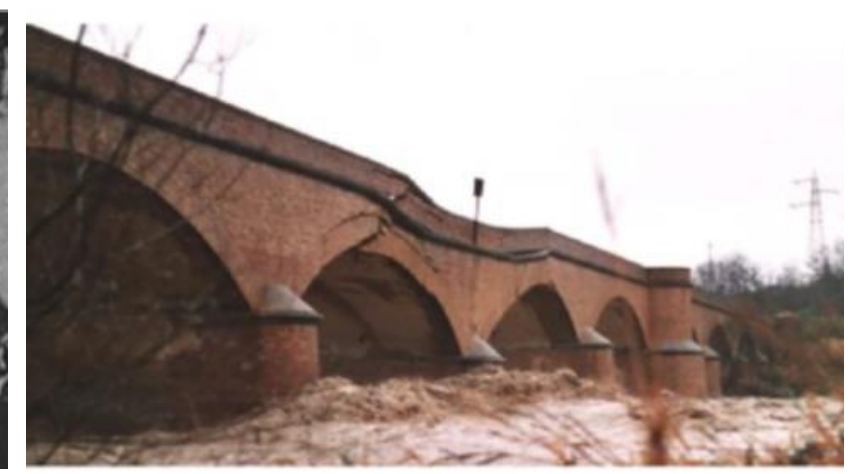

b

Fig. 1: (a) collapse mechanism of Prestwood Bridge (United Kingdom) (Chiozzi et al., 2017); (b) displacement field of Trigno Bridge (Italy) after vertical settlement of the central pier.

However, whether considered as a continuum or as finite number of rigid blocks, when subjected to foundation settlements, masonry structures shall deform like mechanisms in order to be able to accommodate such settlements. In fact, a pattern of cracks appears, making rigid body displacements possible. Hence, the displacement field is piecewise continuous and strong discontinuities (i.e. cracks) appear along lines (for a two-dimensional problem) or surfaces (for a three-dimensional problem), which actually partition the structure into separate blocks of material (see Fig. 1b).

When multiple blocks of material interact through unilateral contact constraints, as it occurs in masonry structures undergoing failure, the theory of variational inequalities (see, e.g., (Fichera, 1972; Kikuchi and Oden, 1988; Panagiotopoulos, 1985)) and the related complementarity problems (see (Facchinei and Pang, 2004)) appear to be a very natural framework in which to carry the study of the mechanical implications of foundation settlements.

In the present paper, we propose a new perspective on the modeling of the influence of foundation settlements in masonry structures with a rigid no-tension material constitutive law. More precisely, for a given masonry structure, we show that it is possible to model in a consistent way the crack pattern and the associated mechanism induced by applied settlements starting from a proper treatment of unilateral contact constraints arising between contiguous blocks, into which the structure is partitioned after settlements have taken place. The proposed approach relies on the development of two variational formulations for the static analysis of multiple rigid masonry bodies with unilateral 
frictionless contact constraints, whose discretization entails the definition of two, mutually dual, linear programming (LP) problems. For this purpose, in Section 2, the paper first reviews the problem of an elastic body in unilateral contact with a rigid obstacle. For the sake of simplicity, only the frictionless contact - the so-called Signorini-Fichera problem - is considered. Specifically, we recall, in the case of a single elastic body, two variational formulations based on the search for extreme points of quadratic forms defined on convex sets. The first is the classical one based on the minimum of the total potential energy; the second, formally obtained by the Green functions of the problem, has, as primal variable, only the contact pressure that the body exchanges with the constraints (Alliney et al., 1990; Kikuchi and Oden, 1988; Wriggers, 2006).

In Section 3, we deal with the problem of unilateral contact between multiple bodies, highlighting the role of rigid body displacements and the possible associated mechanism. In the case of negligible elastic deformations, the quadratic part of the functionals governing the two formulations vanishes and two linear functionals are left, expressing in one case the work of external loads and in the other one the work of constraint reactions for the assigned displacements or gaps at the boundary.

In Section 4, we briefly review the constitutive relations for the Heyman's no-tension rigid masonry material and extend the two boundary variational formulations to describe the crack pattern and the associated mechanism generated in a continuous masonry structure as a consequence of foundation settlements.

In Section 5, we discretize the two formulations by means of two dual LP problems, which account for the possible presence of foundation settlements. In particular, we discuss the deep differences between the classic limit analysis for masonry structures and the two proposed formulations stemming from the contact problem between masonry blocks. Finally, in Section 6 we present some simple but technically relevant examples useful to illustrate the peculiarities of the proposed approach.

\section{Basic relationships for unilateral contact problems}

In this Section we review the variational formulation for a single elastic body in frictionless unilateral contact with a rigid obstacle (i.e. the classical Signorini-Fichera problem, (Fichera, 1964)). In the next Section, we will extend the discussion to the case of multiple bodies in unilateral contact.

\subsection{Boundary variational formulation for a single elastic body in unilateral contact}

We consider a linear hyper-elastic body $\mathcal{B}$ occupying an open, bounded, simply connected domain $\Omega$ in the Euclidean space $\mathbb{R}^{2}$, with a boundary $\Gamma=\partial \Omega$. Let us assume that the body $\mathcal{B}$ is in frictionless unilateral contact with a rigid obstacle whose possible contact surface with $\mathcal{B}$ is denoted by $\Gamma_{c}$ (see Fig. 2), where the Signorini-Fichera boundary conditions hold. The boundary of the body 
$\Gamma$ is assumed to be sufficiently regular and can be decomposed as $\Gamma=\Gamma_{u} \cup \Gamma_{q} \cup \Gamma_{c}$, where $\Gamma_{u}$ and $\Gamma_{q}$ denote the portions of the boundary where essential and natural boundary conditions are respectively applied.

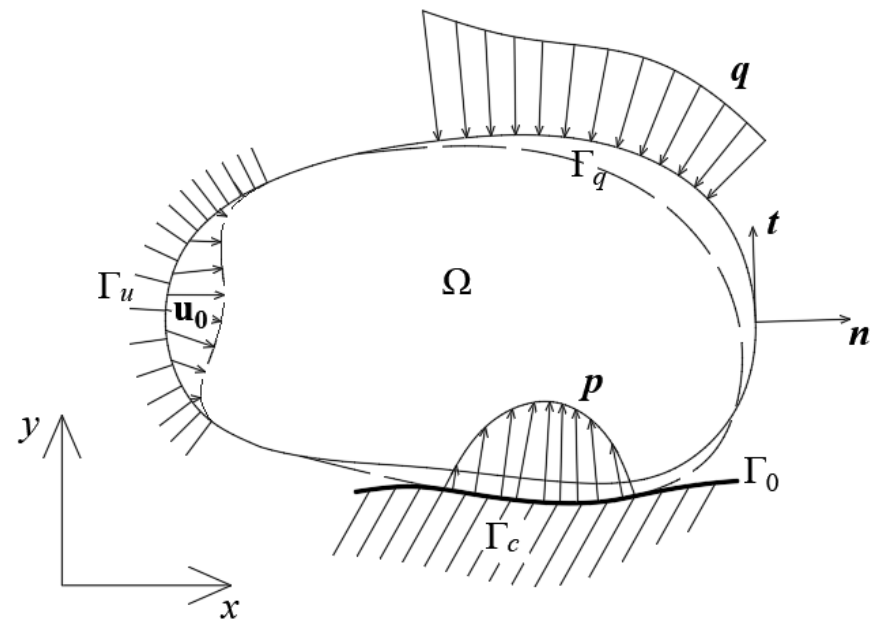

Fig. 2: Two dimensional elastic body in contact with a rigid frictionless obstacle.

In this way a mixed boundary value problem in terms of the displacement field $\boldsymbol{u} \in H^{1}(\Omega)$ can be obtained by assigning the non-homogeneous essential boundary conditions $\boldsymbol{u}=\boldsymbol{u}_{0} \quad$ on $\Gamma_{u}$ and the external loads $\boldsymbol{q}$ on $\Gamma_{q}$. From the mechanics of the problem, it is easy to see that $\Gamma_{c} \cap \Gamma_{u}=\varnothing$. On the other hand, for the sake of simplicity, we assume that $\Gamma_{c} \cap \Gamma_{q}=\varnothing$. We denote also $\boldsymbol{n}$ the outward unit normal vector to $\Gamma$, by $\boldsymbol{\sigma}$ the Cauchy stress tensor and by $\boldsymbol{t}=\boldsymbol{\sigma} \boldsymbol{n}$ the traction vector on the boundary. Let $\boldsymbol{\varepsilon}$ be the infinitesimal strain tensor and $\mathbf{C}$ the Hooke's fourth order elasticity tensor, satisfying the assumptions of symmetry and strong ellipticity, assumed constant all over $\mathcal{B}$. The Signorini-Fichera frictionless boundary conditions read:

$$
\begin{array}{r}
u_{n} \leq 0, t_{n} \leq 0, u_{n} t_{n}=0 \quad \text { on } \Gamma_{c}, \\
t_{t}=0 \text { on } \Gamma_{c}
\end{array}
$$

where $u_{n}$ (resp. $t_{n}$ ) is the normal component of $\boldsymbol{u}$ (resp. $\boldsymbol{t}$ ) with respect to $\Gamma$ and $t_{t}$ is the tangential component of $\boldsymbol{t}$ on $\Gamma$. Further, we have:

$$
\begin{aligned}
& \operatorname{div}(\boldsymbol{\sigma})+\boldsymbol{f}=\mathbf{0} \quad \text { in } \Omega \\
& \boldsymbol{\varepsilon}(\boldsymbol{u})=\frac{1}{2}\left(\nabla \boldsymbol{u}+\nabla \boldsymbol{u}^{T}\right) \quad \text { in } \Omega \\
& \boldsymbol{\sigma}=\mathbf{C} \boldsymbol{\varepsilon} \quad \text { in } \Omega,
\end{aligned}
$$


where $f$ represents the volume force vector. For the sake of simplicity, in the following we assume that $\boldsymbol{f}=\mathbf{0}$ over the whole domain $\Omega$.

Such problem, if we exclude possible rigid motions, has a unique solution which can be found by minimizing the total potential energy functional

$$
\Pi(v)=\frac{1}{2} a(v, v)-\ell(v)
$$

where $a(\cdot, \cdot)$ is a convex, coercive bilinear form and $\ell(\cdot)$ a bounded linear functional which, under the assumption of small displacements and deformations, are respectively defined as:

$$
\begin{aligned}
& a(\boldsymbol{u}, \boldsymbol{v})=\int_{\Omega} \mathbf{C} \boldsymbol{\varepsilon}(\boldsymbol{u}) \boldsymbol{\varepsilon}(\boldsymbol{v}) d \Omega, \\
& \ell(\boldsymbol{v})=\int_{\Gamma_{q}} \boldsymbol{q u} d \gamma
\end{aligned}
$$

The assumption of small displacements and deformations allows assuming no major changes in the contact surface during the deformation process.

Due to the Signorini-Fichera boundary conditions, the minimization of $\Pi(v)$ is to be performed over all the admissible displacements $v \in K$, where $K$ is the convex set defined as follows:

$$
K \equiv\left\{\boldsymbol{v} \in H^{1}(\Omega) ; \boldsymbol{v}=\boldsymbol{u}^{0} \text { on } \Gamma_{u} ; \boldsymbol{v} \cdot \boldsymbol{n}-\Delta(\boldsymbol{x}) \leq \mathbf{0} \text { on } \Gamma_{c}\right\}
$$

where $\Delta(\boldsymbol{x})$ denotes the initial gap, measured along the outer normal, between the undeformed body and the obstacle surface. It can be shown that the solution $\boldsymbol{u}$ of the minimization problem at hand, which can be written as:

$$
\boldsymbol{u}=\arg \inf _{v \in K} \Pi(v)
$$

is also the (unique) solution of the following variational inequality (Fichera, 1972; Panagiotopoulos, 1985):

$$
a(\boldsymbol{u}, \boldsymbol{v}-\boldsymbol{u})-\ell(\boldsymbol{v}-\boldsymbol{u}) \geq 0, \quad \forall \boldsymbol{v} \in K
$$

The variational inequality (10) - or, equivalently, the minimization problem (9) - is the starting point for the finite element solution methods for the problem of an elastic body in unilateral contact with a rigid obstacle without friction (Wriggers, 2006).

An alternative way of tackling the problem is to include the constraint: 


$$
\boldsymbol{v} \cdot \boldsymbol{n}-\Delta(\boldsymbol{x}) \leq 0 \text { on } \Gamma_{c},
$$

by means of a Lagrange multiplier $\lambda(\boldsymbol{x})$. Hence, the problem is reduced to finding a stationary point of the following Lagrangean functional:

$$
\Pi_{\Delta}(\boldsymbol{v}, \lambda)=\frac{1}{2} a(\boldsymbol{v}, \boldsymbol{v})-\ell(\boldsymbol{v})-\int_{\Gamma_{c}} \lambda(\boldsymbol{x})(\boldsymbol{v} \cdot \boldsymbol{n}-\Delta(\boldsymbol{x})) d \gamma
$$

with $v \in U$ and $\lambda \in L$, where $U$ is an affine linear space and $L$ a convex cone respectively defined as:

$$
\begin{gathered}
U \equiv\left\{\boldsymbol{v} \in H^{1}(\Omega) ; \boldsymbol{v}-\boldsymbol{u}^{0}=0 \text { on } \Gamma_{u}\right\}, \\
L \equiv\left\{\lambda \in H^{-1 / 2}\left(\Gamma_{c}\right) ; \lambda \geq 0 \text { on } \Gamma_{c}\right\} .
\end{gathered}
$$

The multiplier $\lambda$ can be mechanically interpreted as the normal, compression-only, constraint reaction along $\Gamma_{c} . \boldsymbol{u}$ is a solution of the mixed variational problem arising from the minimization of (12) if and only if there exist $p \in L$ such that $(\boldsymbol{u}, p)$ is a saddle point of $\Pi_{\Delta}$ on $U \times L$, i.e. if and only if:

$$
\Pi_{\Delta}(\boldsymbol{u}, p)=\inf _{\boldsymbol{v} \in U}\left(\sup _{\lambda \in L} \Pi_{\Delta}(\boldsymbol{v}, \lambda)\right)=\sup _{\lambda \in L}\left(\inf _{\boldsymbol{v} \in U} \Pi_{\Delta}(\boldsymbol{v}, \lambda)\right) .
$$

Therefore, finding the solution $(\boldsymbol{u}, p)$ entails either solving the following minimization problem, known as primal problem, in terms of displacements:

$$
\boldsymbol{u}=\arg \inf _{\boldsymbol{v} \in U}\left(\sup _{\lambda \in L} \Pi_{\Delta}(\boldsymbol{v}, \lambda)\right)
$$

or solving the following maximization problem, known as dual problem, in terms of tractions:

$$
p=\arg \sup _{\lambda \in L}\left(\inf _{v \in U} \Pi_{\Delta}(v, \lambda)\right)
$$

For our purposes, it is now useful to introduce a variational formulation for the problem at hand defined only on the boundary $\Gamma$ of the body $\mathcal{B}$ (see, e.g., (Alliney et al., 1990; Antes and Panagiotopoulos, 1992)). To this aim, let $\mathbf{G}(\boldsymbol{x}, \boldsymbol{\xi}), \boldsymbol{x} \in \Omega, \boldsymbol{\xi} \in \Gamma$, be the Green's operator, related to the homogeneous boundary conditions $\boldsymbol{u}=\mathbf{0}$ on $\Gamma_{u}$ and $\boldsymbol{q}=\mathbf{0}$ on $\Gamma_{q}$. Let us recall that the $i, j$-th element $G_{i j}(\boldsymbol{x}, \boldsymbol{\xi})$ of matrix $\mathbf{G}$, represents the $i$-th component of the displacement vector at point $\boldsymbol{\xi}$ 
due to a unit-force at source point $\boldsymbol{x}$ acting in the direction $x_{j}$. Analogously, let $\boldsymbol{H}(\boldsymbol{x}, \boldsymbol{\xi})$ be the matrix, associated to $\mathbf{G}$, whose $i, j$-th element $H_{i j}(\boldsymbol{x}, \boldsymbol{\xi})$, is the $i$-th component of the traction vector at point $\xi$ due to a unit-force at source point $\boldsymbol{x}$ acting in the direction $x_{j}$. Since frictionless contact is assumed, then, for any normal reaction vector $\boldsymbol{n}(\xi) \lambda(\xi)$ on $\Gamma_{c}$, load vector $\boldsymbol{q}(\boldsymbol{\xi})$ on $\Gamma_{q}$ and assigned displacements $\boldsymbol{u}_{0}(\boldsymbol{\xi})$ on $\Gamma_{u}$, the induced displacement field over $\mathcal{B}$ can be computed as follows (Antes and Panagiotopoulos, 1992):

$$
\boldsymbol{v}_{\lambda}(\boldsymbol{x})=\int_{\Gamma_{c}} \mathbf{G}(\boldsymbol{x}, \boldsymbol{\xi}) \lambda(\boldsymbol{\xi}) \boldsymbol{n}(\boldsymbol{\xi}) d \Gamma(\boldsymbol{\xi})+\int_{\Gamma_{q}} \mathbf{G}(\boldsymbol{x}, \boldsymbol{\xi}) \boldsymbol{q}(\boldsymbol{\xi}) d \Gamma(\boldsymbol{\xi})-\int_{\Gamma_{u}} \mathbf{H}(\boldsymbol{x}, \boldsymbol{\xi}) \boldsymbol{u}_{0}(\boldsymbol{\xi}) d \Gamma(\boldsymbol{\xi})
$$

where $\boldsymbol{v} \in U$. It should be observed that, if $\boldsymbol{x} \in \Gamma_{u}$, then:

$$
\begin{cases}\mathbf{H}(x, \xi)=\mathbf{I} & \text { if } \boldsymbol{\xi} \equiv \boldsymbol{x} \\ \mathbf{H}(\boldsymbol{x}, \boldsymbol{\xi})=\mathbf{0} & \text { otherwise }\end{cases}
$$

where $\mathbf{I}$ is the identity matrix.

It is now possible to obtain a new Lagrangean functional equivalent to (12) but entirely defined on the boundary of $\mathcal{B}$. To this end, it should be pointed out that the presence of non-homogeneous essential conditions $\boldsymbol{u}=\boldsymbol{u}_{0}$ on $\Gamma_{u}$ implies that an additional Lagrange multiplier $\rho(\boldsymbol{x}) \in R$, where $R \equiv H^{-1 / 2}\left(\Gamma_{u}\right)$, must be introduced in the boundary formulation, in order to account for the presence of constraint reactions along $\Gamma_{u}$, directed as $\boldsymbol{u}_{0}$.

Substituting (18) into (12), recalling (19) and equilibrium equations (3), and applying the GaussGreen identity we obtain the following functional in terms of $\lambda, \rho$ only:

$$
\Pi_{\Delta}^{*}(\lambda, \rho)=\Pi_{\Delta}\left(v_{\lambda}, \lambda\right)=-\frac{1}{2} a^{*}(\lambda, \lambda)+\ell^{*}(\lambda)+c_{u}(\rho)+c_{q}
$$

where:

$$
\begin{aligned}
& a^{*}(\lambda, \lambda)=\int_{\Gamma_{c}} \int_{\Gamma_{c}} \lambda(\boldsymbol{x}) \boldsymbol{n}(\boldsymbol{x}) \mathbf{G}(\boldsymbol{x}, \boldsymbol{\xi}) \lambda(\boldsymbol{\xi}) \boldsymbol{n}(\boldsymbol{\xi}) d \Gamma(\boldsymbol{x}) d \Gamma(\boldsymbol{\xi}), \quad \boldsymbol{x}, \boldsymbol{\xi} \in \Gamma_{c} \\
& \ell^{*}(\lambda)=-\int_{\Gamma_{c}} \int_{\Gamma_{q}} \lambda(\boldsymbol{x}) \boldsymbol{n}(\boldsymbol{x}) \mathbf{G}(\boldsymbol{x}, \boldsymbol{\xi}) \boldsymbol{q}(\boldsymbol{\xi}) d \Gamma(\boldsymbol{x}) d \Gamma(\boldsymbol{\xi})+\int_{\Gamma_{c}} \lambda(\boldsymbol{x}) \Delta(\boldsymbol{x}) d \Gamma(\boldsymbol{x}), \quad \boldsymbol{x} \in \Gamma_{c}, \quad \boldsymbol{\xi} \in \Gamma_{q} \\
& c_{u}(\rho)=\frac{1}{2} \int_{\Gamma_{u}} \rho(\xi) \boldsymbol{u}^{0}(\xi) d \Gamma(\xi), \quad \boldsymbol{\xi} \in \Gamma_{u}
\end{aligned}
$$




$$
c_{q}=-\frac{1}{2} \int_{\Gamma_{q}} \int_{\Gamma_{q}} \boldsymbol{q}(\boldsymbol{x}) \mathbf{G}(\boldsymbol{x}, \boldsymbol{\xi}) \boldsymbol{q}(\boldsymbol{\xi}) d \Gamma(\boldsymbol{x}) d \Gamma(\boldsymbol{\xi}), \quad \boldsymbol{x}, \boldsymbol{\xi} \in \Gamma_{q} .
$$

The symmetry of the Green's matrix $\mathbf{G}$ with respect to the arguments guarantees the symmetry of the bilinear form $a^{*}(\cdot, \cdot)$. Moreover, the bilinear form $a^{*}(\cdot, \cdot)$ preserves ellipticity and coerciveness (Kikuchi and Oden, 1988). Thus, the boundary tractions $(p, r)$ on both $\Gamma_{c}$ and $\Gamma_{u}$, are obtained by solving the following minimization problem:

$$
(p, r)=\arg \inf _{\lambda \in L, \rho \in R}\left(\Pi_{\Delta}^{*}(\lambda, \rho)\right)=\arg \inf _{\lambda \in L, \rho \in R}\left(-\frac{1}{2} a^{*}(\lambda, \lambda)+\ell^{*}(\lambda)+c_{u}(\rho)\right) .
$$

We refer to (Kikuchi and Oden, 1988; Wriggers, 2006) for the discussion of different and more complex contact conditions. However, we recall that, for the contact with friction, these classical variational formulations are not possible. Observe that if $\mathcal{B}$ is not sufficiently constrained and rigid body motions are allowed, then the solution is not unique.

\section{Boundary variational formulation for multiple elastic bodies in unilateral contact}

\subsection{Unilateral contact between multiple elastic bodies}

Let us consider $N_{b}$ linear hyper-elastic bodies $\mathcal{B}_{i}\left(i=1, \ldots, N_{b}\right)$ respectively occupying the open, bounded, simply connected domains $\Omega_{i} \subset \mathbb{R}^{2}$, whose boundaries $\Gamma_{i}$ are sufficiently regular (Fig. 3a).

Let us define $\Omega=\bigcup_{i=1}^{N_{b}} \Omega_{i}$. Moreover, we denote by $\Gamma_{c}$ the union of all the $N_{c}$ surfaces of possible contact between the $N_{b}$ bodies, whereas we denote by $\Gamma_{u}$ the union of all the surfaces belonging to the $N_{b}$ bodies subjected to the essential boundary conditions $\boldsymbol{u}=\boldsymbol{u}_{0}$.

When multiple bodies in mutual unilateral contact are considered, rigid body motions are generally possible. In this case, the displacement field $\boldsymbol{u}(\boldsymbol{x}), \boldsymbol{x} \in \Omega$, is only piecewise continuous and can be assumed of special bounded variation, i.e. $\boldsymbol{u} \in S B V(\Omega)$, see (Ambrosio and De Giorgi, 1988). Be $M$ the set of all the admissible, piecewise continuous, displacement fields $\boldsymbol{u}$. In $M$ can exist displacement fields that do not present elastic deformations: such displacement fields are called mechanisms. The set of all these mechanisms is denoted as $M^{*}, M^{*} \subset M$.

In order to define the possible mechanism arising from a constraint settlement, we have to clarify the contact conditions. Let us denote by $\boldsymbol{t}, \boldsymbol{n}$ the unit tangent and the unit normal to $\Gamma_{c}$ respectively. Call $\boldsymbol{u}^{+}$and $\boldsymbol{u}^{-}$the value of the displacement field on the two sides of the curve $\Gamma_{c}, \boldsymbol{u}^{+}$being the 
displacement in the portion of the domain $\Omega$ toward which $\boldsymbol{n}$ points. The jump of $\boldsymbol{u}$ across $\Gamma_{c}$ can be denoted as follows:

$$
\boldsymbol{u}=\boldsymbol{u}^{+}-\boldsymbol{u}^{-}
$$

and decomposed into tangential and normal components:

$$
\boldsymbol{u}=u_{t} \boldsymbol{t}+u_{n} \boldsymbol{n}, u_{t}=\boldsymbol{u} \cdot \boldsymbol{t}, u_{n}=\boldsymbol{u} \cdot \boldsymbol{n} .
$$

In the case of frictionless contact $u_{n} \geq 0$ while $u_{t}$ is not constrained. However, different contact conditions have to be introduced in the following for Heyman masonry-like material.

Let us remark that the possible existence of mechanisms in $\Omega$ is obviously predicted also by the theory of limit analysis for masonry structures (Como, 2017, 1992). Therefore, focusing on masonry structures, we may assume from the beginning the presence of a finite number of $N_{b}$ bodies into which a set of cracks completely partitions the original structure. The $N_{b}$ bodies are mutually interacting by means of unilateral constraint conditions. In general, due to the opening of cracks, the displacement field is only piecewise continuous and has a strong discontinuity on a finite number of curves in $\Gamma_{c}$ (Figs. 3a-b).

Moreover, when $N_{b}$ elastic bodies in unilateral contact are considered, the displacement field $\boldsymbol{u}$ belongs to a convex set $M$ in a special bounded variation function space, precisely:

$$
M \equiv\left\{\boldsymbol{v} \in \operatorname{SBV}\left(\Omega_{i}\right), i=1, \ldots, n ; \boldsymbol{v}=\boldsymbol{u}^{0} \text { on } \Gamma_{u} ; v_{n} \leq \Delta(\boldsymbol{x}) \text { on } \Gamma_{c}\right\}
$$

$\Delta(\boldsymbol{x})$ and $v_{n}=\boldsymbol{v} \cdot \boldsymbol{n}$ denote respectively the mutual gap and the mutual displacements in the direction normal to the possible contact curve between the bodies. Finally, possible rigid body motions $\boldsymbol{u}_{r}$ belong to the set $M^{*}$ defined as:

$$
M^{*} \equiv\left\{v \in M ; \nabla \boldsymbol{v}^{T}=-\nabla \boldsymbol{v}\right\} .
$$

The solution of the problem, in analogy with (9), is given by the field $\overline{\boldsymbol{u}}$ obtained from the following minimization:

$$
\boldsymbol{u}=\arg \inf _{v \in M} \Pi(v)
$$

where:

$$
\Pi(v)=\sum_{i=1}^{N_{b}}\left(\frac{1}{2} a_{i}(v, v)-\ell_{i}(v)\right)
$$


is the total potential energy functional for the set of $N_{b}$ bodies. In (31) we denote as $a_{i}(\cdot, \cdot)$ and $\ell_{i}(\cdot)$ the bilinear and linear forms (7) defined on each subdomain $\Omega_{i}$.

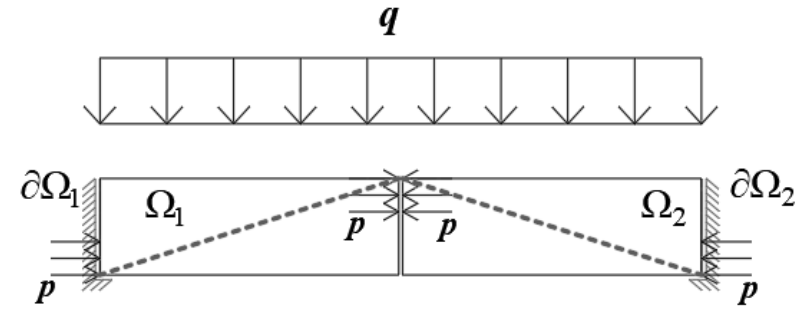

a

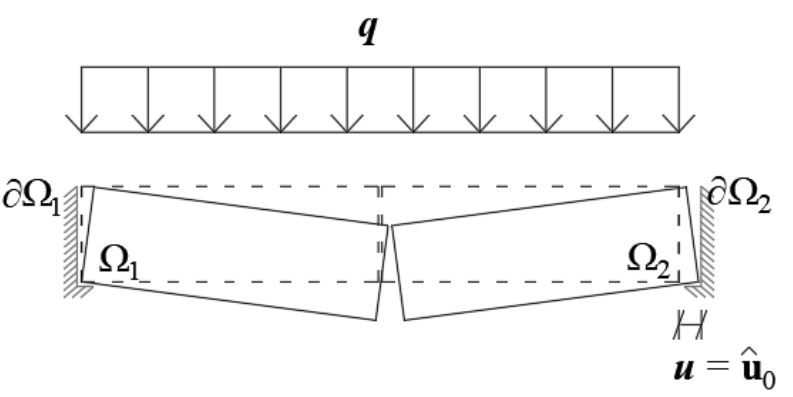

b

Fig. 3: (a-b) Unilateral contact between multiple elastic bodies with homogeneous and nonhomogeneous essential boundary conditions, respecively.

Let us remark that in (31) the bilinear form is symmetric and semi coercive and the linear form can be unbounded due to the possible presence of mechanisms belonging to $M^{*}$, but is bounded if we assume rigid body motions of finite amplitude.

Analogously to (25), the boundary tractions $(p, r)$ on both $\Gamma_{c}$ and $\Gamma_{u}$ respectively are obtained by solving the following boundary minimization problem:

$$
(p, r)=\arg \inf _{\lambda \in L, \rho \in R}\left(\Pi_{\Delta}^{*}(\lambda, \rho)\right)=\arg \inf _{\lambda \in L, \rho \in R}\left(\sum_{i=1}^{N_{b}}-\frac{1}{2} a_{i}^{*}(\lambda, \lambda)+\ell_{i}^{*}(\lambda)+c_{u, i}(\rho)\right),
$$

where the set $R$ has been defined in the previous Subsection. Since, in order to guarantee the uniqueness of the solution, each body $\mathcal{B}_{i}$ must be in global equilibrium under the action of external forces and contact tractions, the following new definition for the set $L$ is provided:

$$
L \equiv\left\{\lambda \in H^{-1 / 2}\left(\Gamma_{c}\right) ; \lambda \geq 0 \text { on } \Gamma_{c} ; \lambda \text { s.t. } \mathcal{A}_{i}(\lambda, \rho, \boldsymbol{q})=0, i=1, \ldots, N_{b}\right\}
$$

where the operator $\mathcal{A}_{i}$ represents the three global equilibrium conditions (two translational and one rotational) for each body $\mathcal{B}_{i}$.

\subsection{Unilateral contact between multiple rigid bodies}

Let us now consider structures composed of a finite number of rigid blocks, which cannot fracture within their interior.

Constitutive relations for rigid bodies can be derived following for instance the classical approach proposed by Grioli (Grioli, 1983), who defined a rigid body as the limit of a hyper-elastic body when 
its elastic stiffness tends to infinity. In the present work, we will restrict Grioli's theory to static problems and linear isotropic bodies. Then, the rigid body is obtained in the limit by making the Young modulus $E$ tend to infinity, and is characterized by the condition:

$$
\lim _{E \rightarrow \infty} \varepsilon=0
$$

Moreover, as observed in (Iannuzzo et al., 2018), a recent piecewise rigidity result in (Chambolle et al., 2007) generalizing the classical Liouville result for smooth functions, states that if the displacement field $\boldsymbol{u}$ in a cracked body is a function of special bounded variation on $\Omega$, i.e. $u \in \operatorname{SBV}(\Omega)$, such that its gradient is almost everywhere a rotation, i.e. $\nabla \boldsymbol{u} \in \mathrm{SO}(2)$, then $\boldsymbol{u}$ is a collection of an at most countable family of rigid motions. In other words, the cracked body does not store elastic energy if and only if all its connected components are deformed through rigid motions. Therefore, in the case of multiple rigid bodies in mutual contact, the bilinear part in (31) vanishes and the solution $\boldsymbol{u}$ of the variational contact problem is given by

$$
\boldsymbol{u}=\arg \inf _{\boldsymbol{v} \in M^{*}} \Pi_{r}(\boldsymbol{v}),
$$

where

$$
\Pi_{r}(v)=-\sum_{i=1}^{N_{b}} \ell_{i}(v)
$$

is the potential energy of external loads and $M^{*}$ has been defined in (29).

Thus, a displacement field which is the solution of the boundary value problem, can be found by minimizing the potential energy of the external loads in the set $\boldsymbol{u} \in M^{*}$ containing all the possible mechanisms which satisfy the unilateral constraints.

Let us observe that, since the deformation field has a jump discontinuity along the regular curve $\Gamma_{c}$, then also the stress field $\boldsymbol{p}=\boldsymbol{\sigma} \boldsymbol{n}, \boldsymbol{\sigma}$ being the Cauchy stress tensor, exhibits a jump discontinuity across $\Gamma_{c}$ and on such curve the stress can be defined as a line Dirac delta function whose amplitude is related to the jump of $\boldsymbol{p}$ across $\Gamma_{c}$ (Lucchesi et al., 2006; Šilhavý, 2014). Therefore, for rigid bodies the definition for the admissible set of boundary pressures given in (14) does not hold anymore. Let us denote the jump of the stress field $\boldsymbol{p}$ across $\Gamma_{c}$ as

$$
p=p^{+}-p^{-}
$$


where $\boldsymbol{p}^{+}$and $\boldsymbol{p}^{-}$are the value of the stress field on the two sides of the curve $\Gamma_{c}, \boldsymbol{p}^{+}$being the stress in the portion of the domain $\Omega$ toward which $\boldsymbol{n}$ points. Moreover, the jump of $\boldsymbol{p}$ across $\Gamma_{c}$ can be decomposed into normal and tangential components:

$$
\boldsymbol{p}=p_{t} \boldsymbol{t}+p_{n} \boldsymbol{n}, \quad p_{t}=\boldsymbol{p} \cdot \boldsymbol{t}, \quad p_{n}=\boldsymbol{p} \cdot \boldsymbol{n} .
$$

In the frictionless Signorini contact problem the following constraint relations hold:

$$
p_{n} \leq 0, p_{t}=0
$$

Hence, the set of the admissible boundary pressures for an assemblage of rigid bodies in mutual contact can be redefined as follows as the set $L^{*}$ :

$$
L^{*} \equiv\left\{\boldsymbol{p} \in D^{\prime}(\Gamma) ; p_{n} \leq 0 \text { on } \Gamma_{c} ; p_{t}=0 \text { on } \Gamma_{c} ; p_{n} \text { s.t. } \mathcal{A}_{i}\left(p_{n}, \rho, \boldsymbol{q}\right)=0, i=1, \ldots, N_{b}\right\}
$$

where $D^{\prime}$ is the dual of the space of test functions on $\Gamma$, i.e. the space of distributions on $\Gamma$. Moreover, in the problem at hand, the rigid body assumption modifies the complementary functional as well, since it holds:

$$
\lim _{E \rightarrow \infty} \mathbf{G}(\boldsymbol{x}, \boldsymbol{\xi})=\mathbf{0}
$$

Therefore, the bilinear part (21) vanishes and the linear part (22) reduces to

$$
\ell^{* r}(\lambda)=\int_{\Gamma_{c}} \lambda(\boldsymbol{x}) \Delta(\boldsymbol{x}) d \gamma, \quad \boldsymbol{x} \in \Gamma_{c} .
$$

Then, the boundary complementary problem in terms of boundary tractions is stated as follows:

$$
(p, r)=\arg \inf _{\lambda \in L^{*}, \rho \in R}\left(\Pi_{\Delta}^{* r}(\lambda, \rho)\right)
$$

where

$$
\Pi_{\Delta}^{* r}(\lambda, \rho)=\sum_{i=1}^{N_{b}} \ell_{i}^{* r}(\lambda)+c_{u, i}(\rho),
$$

In other words, the distribution of unilateral contact pressures can be found by minimizing the work of contact pressures for possible gaps and constraint reactions for the assigned displacements $\boldsymbol{u}^{0}$. The complementary boundary variational principle (43) for rigid bodies appears new in the literature. 


\section{Continuous masonry structures}

In Section 3.2, we accounted for only one of Heyman's hypotheses, i.e. the assumption of negligible elastic deformations. However, in order to deal with masonry material, it is also necessary to take into account the following assumptions:

- Masonry is incapable of withstanding tensions,

- Masonry has infinite compressive strength

- Sliding cannot occur because masonry has infinite shear strength.

We define a masonry-like material as a continuum that is Rigid and No-Tension (RNT, see (Angelillo, 2014)) in the sense defined by previous assumptions. In this framework, in the last decades, several Authors, especially Italian, studied the equilibrium analysis of masonry structures using $S B V$ functional spaces for displacement fields of various kind, (see, e.g., (Del Piero, 1998)). In the following, the problem is re-discussed. Specifically, we refer to recent papers by M. Como and M. Angelillo and co-authors (see, e.g., (Angelillo, 1993, 2014; Angelillo et al., 2014; Como, 1992, 2013)), which dedicate particular attention to the treatment of discontinuities in the displacement field and to the consequent singularities arising in stress and deformation fields.

\subsection{Constitutive restrictions on strain and stress for masonry material}

In a no-tension material, when the compression stresses holding stones together vanish in some regions of the body, cracks appear and the displacements field is not continuous anymore. However, the displacement field does not admit elastic contractions and, therefore, it is generally associated to a mechanism. As in the cases previously discussed, when cracks open we can define the new boundary $\Gamma_{c}$ of the cracked body that includes all the fracture lines associated to the given displacement field $\boldsymbol{u}(\boldsymbol{x})$. Moreover, since the displacement field is a mechanism (i.e. $\nabla \boldsymbol{u}^{T}=-\nabla \boldsymbol{u}$.), each body $\mathcal{B}_{i}$ can only undergo rigid rotation and translation motions.

For a RNT material the following restrictions hold (see, for example, (Iannuzzo et al., 2018)):

$$
\boldsymbol{\sigma} \in S^{-} m^{-}, \boldsymbol{\varepsilon} \in \operatorname{Sym}^{+}, \boldsymbol{\sigma} \cdot \boldsymbol{\varepsilon}=0,
$$

where $\mathrm{Sym}^{-}$and $\mathrm{Sym}^{+}$represent the convex cones of negative semidefinite and positive semidefinite symmetric tensors respectively.

Under these restrictions, the material satisfies a law of normality with respect to the cone $\mathrm{Sym}^{-}$of the feasible stresses, in the sense that restrictions (45) are equivalent to the following normality assumptions:

$$
\boldsymbol{\sigma} \in S y m^{-},\left(\boldsymbol{\sigma}-\boldsymbol{\sigma}^{*}\right) \cdot \boldsymbol{\varepsilon} \geq 0, \forall \boldsymbol{\sigma}^{*} \in S y m^{-},
$$


and to the dual normality assumptions:

$$
\boldsymbol{\varepsilon} \in \operatorname{Sym}^{+},\left(\boldsymbol{\varepsilon}-\boldsymbol{\varepsilon}^{*}\right) \cdot \boldsymbol{\sigma} \geq 0, \forall \boldsymbol{\varepsilon}^{*} \in S y m^{+} .
$$

These normality assumptions represent the essential ingredients for proving the validity of the theorems of limit analysis (see, e.g., (Del Piero, 1998)). Restrictions (46) and (47) actually formalize in mathematical terms Heyman's assumptions for masonry material.

\subsection{Singular strain and stress}

For RNT materials, it is possible to admit that strain and stress are bounded measures. Bounded measures can be decomposed into the sum of two parts:

$$
\boldsymbol{\varepsilon}=\boldsymbol{\varepsilon}^{c}+\boldsymbol{\varepsilon}^{s}, \boldsymbol{\sigma}=\boldsymbol{\sigma}^{c}+\boldsymbol{\sigma}^{s}
$$

where $(\cdot)^{c}$ is the part that is absolutely continuous with respect to the area measure (in other words $(\cdot)^{c}$ is a density per unit area) and $(\cdot)^{s}$ is the singular part. On admitting singular strains and stresses, it is possible to assume that both the displacement $\boldsymbol{u}$ and the stress vector $\boldsymbol{p}$ are discontinuous, see (38). It is true also for RNT materials that if the displacement field exhibits a jump discontinuity across a regular curve $\Gamma$, on such a curve the associated strain is a line Dirac delta function $\delta_{\Gamma}(\boldsymbol{x})$, whose amplitude coincides with the value of the jump of $\boldsymbol{u}$ along $\Gamma$ (see Fig.4).

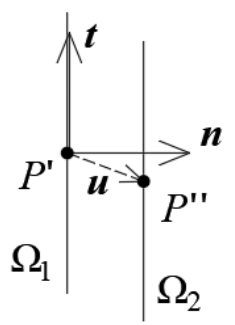

$$
\begin{aligned}
& u_{n}=\boldsymbol{u} \cdot \boldsymbol{n} \geq 0 \\
& u_{t}=\boldsymbol{u} \cdot \boldsymbol{t} \text { free }
\end{aligned}
$$

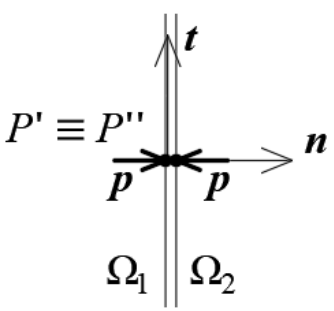

$$
\begin{aligned}
& p_{n}=|\boldsymbol{p}| \cdot \boldsymbol{n} \leq 0 \\
& p_{t}=|\boldsymbol{p}| \cdot \boldsymbol{t}=0
\end{aligned}
$$

a

b

Fig. 4: Kinematic interface conditions for the Signorini contact problem between elastic bodies.

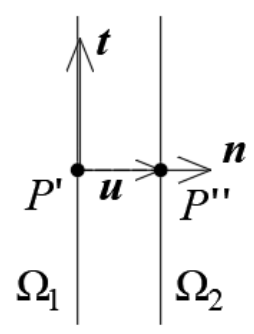

$$
\begin{aligned}
& u_{n}=\boldsymbol{u} \cdot \boldsymbol{n} \geq 0 \\
& u_{t}=\boldsymbol{u} \cdot \boldsymbol{t}=0
\end{aligned}
$$$$
P^{\prime} \equiv P^{\prime \prime} \underbrace{}_{\Omega_{1}}
$$

$$
\begin{aligned}
& p_{n}=|\boldsymbol{p}| \cdot \boldsymbol{n} \leq 0 \\
& p_{t}=|\boldsymbol{p}| \cdot \boldsymbol{t} \text { free }
\end{aligned}
$$

a

Fig. 5: Kinematic interface conditions for the contact problem between rigid no-tension masonry like bodies (Heyman's hypotheses). 
Therefore, it is possible to admit that material restrictions on strains take the form:

$$
\boldsymbol{\varepsilon}=u_{n} \delta_{\Gamma}(\boldsymbol{x}) \boldsymbol{n} \otimes \boldsymbol{n}, u_{n} \geq 0
$$

Moreover, taking into account the restriction $\boldsymbol{\varepsilon} \in \mathrm{Sym}^{+}$, it must be:

$$
u_{t}=0
$$

That is, the two portions $\Omega_{i}$ and $\Omega_{i+1}$ bordering along $\Gamma_{c}$ may separate but cannot penetrate each other, and the sliding component $u_{t}$ along $\Gamma_{c}$ must be zero, in agreement with Heyman hypotheses. For the sake of simplicity, let us neglect any crack inside the generic block associated to a given mechanism and assume that all the discontinuities in the displacement field are on the boundary $\Gamma_{c}$ separating adjacent bodies. Recalling the restrictions introduced above and (38) the concentrated stress $\boldsymbol{\sigma}$ across $\Gamma_{c}$ can be denoted as follows (Lucchesi et al., 2006):

$$
\boldsymbol{\sigma}=P \delta_{\Gamma_{c}}(\boldsymbol{x}) \boldsymbol{t} \otimes \boldsymbol{t}, P^{\prime}+p_{n}=0, P \rho+p_{t}=0, P \leq 0,
$$

where $P$ is the amplitude of the line Dirac delta function along $\Gamma_{c}$, i.e. of the concentrated stress acting along $\Gamma_{c}, \rho$ is the curvature of the curve $\Gamma_{c}$, and $P^{\prime}$ is the derivative of $P$ with respect to its argument. The last relation implies that the stress $\boldsymbol{\sigma}$ must be compressive (Lucchesi et al., 2006). In synthesis, at the interface between two masonry blocks one has:

$$
u_{n} \geq 0, p_{n} \leq 0, u_{t}=0
$$

whereas $p_{t}$ is free.

The set of all the admissible mechanisms $M^{* m}$ in this context can be defined by modifying (29) as follows:

$$
M^{* m} \equiv\left\{\boldsymbol{v} \in S B V\left(\Omega_{i}\right), i=1, \ldots, n ; \nabla \boldsymbol{v}^{T}=-\nabla \boldsymbol{v} ; \boldsymbol{v}=\boldsymbol{v}^{0} \text { on } \Gamma_{u} ; v_{n} \leq 0, v_{t}=0 \text { on } \Gamma_{c}\right\} .
$$

where $v_{n}=\boldsymbol{v} \cdot \boldsymbol{n}$ denotes, as previously defined in (27), the mutual rigid displacements in the direction normal to the possible contact line between the bodies.

The set of all admissible contact pressures $L^{* m}$ now contains the tangential components as well, and can be obtained modifying (40) as follows:

$$
L^{* m} \equiv\left\{\boldsymbol{p} \in D^{\prime}\left(\Gamma_{c}\right) ; p_{n} \leq 0 \text { on } \Gamma_{c} ; p_{n} \text { s.t. } \mathcal{A}_{i}\left(p_{n}, \rho, \boldsymbol{q}\right)=0, i=1, \ldots, N_{b}\right\}
$$


where $D^{\prime}$ is the dual of the space of test functions on $\Gamma_{c}$, i.e. the space of distributions on $\Gamma_{c}$.

\subsection{Boundary variational formulation for blocks of masonry}

Also in the case of a system of masonry blocks in mutual contact, the bilinear part of the energy functional vanishes. Hence, the solution of the primal problem is obtained through the following minimization:

$$
\boldsymbol{u}=\arg \inf _{\boldsymbol{v} \in M^{*} m} \Pi_{r}(\boldsymbol{v})
$$

where

$$
\Pi_{r}(v)=-\sum_{i=1}^{N_{b}} \ell_{i}(v)
$$

and $M^{* m}$ is defined in (53). In other words, a displacement field which is the solution of the boundary value problem at hand, can be found by minimizing the potential energy of the external loads in the set $M^{* m}$ of all the mechanisms satisfying the unilateral constraints in the normal direction and the nosliding condition.

The result described is not completely new, see for instance (Angelillo et al., 2018; Iannuzzo et al., 2018), but has been derived in a different way. In fact, the proposed approach allows unveiling a new boundary variational formulation for contact pressures between masonry blocks, which, on the other hand, is new in the literature. Indeed, in an analogous way, the boundary complementary problem in terms of boundary tractions can be written as follows:

$$
(p, r)=\arg \inf _{\lambda \in L^{* m}, \rho \in R}\left(\Pi_{\Delta}^{* r}(\lambda, \rho)\right),
$$

where

$$
\Pi_{\Delta}^{* r}(\lambda, \rho)=\sum_{i=1}^{N_{b}} \ell_{i}^{* r}(\lambda)+c_{u, i}(\rho)
$$

For the sake of simplicity, let us assume that no initial gap $\Delta(\boldsymbol{x})$ is present between masonry blocks. In this case, (58) further simplifies to:

$$
\Pi_{\Delta}^{* r}(\lambda, \rho)=\sum_{i=1}^{N_{b}} c_{u, i}(\rho) .
$$

In other words, in presence of foundation settlements only, the distribution of boundary contact forces can be found by minimizing the work of constraint reactions for the assigned displacements. The definition of the set $L^{* m}$ of possible contact forces at interfaces and boundary reactions given in (54) is defined in a different way from the classic Signorini frictionless contact problem and allows for 
non-constrained in sign contact tangential pressure distributions. The interest in this formulation lays also in the fact that, from the discretization of the two previously illustrated principles, two dual linear programming formulations can be directly derived.

Remark 1. It should be pointed out that, when changing the loading conditions, the cracks previously induced by the settlements must close before contact actions can be newly transferred. For this reason, masonry does not behave like a rigid perfectly plastic material, but as a nonlinear elastic material.

Remark 2. Usually, external constraints are not sufficient to guarantee global equilibrium of the system of rigid blocks. Therefore, equilibrium equations for each block involving both unknown boundary tractions $(\lambda, \rho)$ and external loads $\boldsymbol{q}$ must be imposed as additional constraints on the minimization problem (57).

\section{Discretization and linear programming formulations of the problem}

As we have shown in the previous Section, two minimum problems hold for a set of masonry rigid bodies fulfilling Heyman's hypotheses, laying in mutual contact and undergoing foundation settlements: problem (55), in terms of displacements on the whole domain $\Omega$, and problem (57), in terms of tractions on the part of boundary $\Gamma_{c} \cup \Gamma_{u}$, subjected to both unilateral contact conditions and constraint reactions. The first problem has been derived from the principle of minimization of total potential energy functional, whereas the second has been derived from an appropriate Lagrangean boundary formulation. Obviously, the same methods can be applied to a masonry structure occupying the domain $\Omega$, discretized into a finite number $N_{b}$ of rigid bodies $\mathcal{B}_{i}$, each occupying the subdomain $\Omega_{i} \subset \Omega$, for which Heyman's hypotheses hold, and minimum principles analogous to (55) and (57) can be formulated as linear programming (LP) problems. Let us assume that each possible contact interface belonging to $\Gamma_{c}$, where contact conditions hold, and each externally constrained curve belonging to $\Gamma_{u}$, where essential boundary conditions are imposed, are discretized into an assigned number of collocation points. Be $N_{c o l, p}$ the total number of collocation points on all the interfaces belonging to $\Gamma_{c}$ and $N_{c o l, r}$ the total number of collocation points on all the interfaces belonging to $\Gamma_{u}$.

Let us consider first the minimum problem (55), here reported for the sake of clarity: 


$$
\boldsymbol{u}=\arg \inf _{\boldsymbol{v} \in M^{* m}}(\Pi(\boldsymbol{v}))=\arg \inf _{\boldsymbol{v} \in M^{*} m}\left(-\sum_{i=1}^{N_{b}} \ell_{i}(\boldsymbol{v})\right)
$$

A generic piecewise two-dimensional rigid displacement $v \in M^{*_{m}}$ is represented by a vector $\mathbf{d}$ composed by $3 N_{b}$ elements being the three barycentric components of rigid body motion (two translations and a rotation) for each block. Obviously, it is possible to define the potential energy associated to the rigid body motion $\boldsymbol{v}$ represented by the vector $\mathbf{d}$ as a linear function of $\mathbf{d}$ :

$$
\Pi(v)=-\hat{\mathbf{q}}^{T} \mathbf{d},
$$

where $\hat{\mathbf{q}} \in \mathbb{R}^{3 N_{b}}$ is a vector containing, for each body $\mathcal{B}_{i}$, the resultants - in terms of two translational forces and one moment - of the external load distribution $\boldsymbol{q}(\boldsymbol{x})$ on $\Gamma_{q}$. Therefore, the discrete LP problem associated to (60) can be written as follows:

$$
\text { find d s.t. }\left\{\hat{\mathbf{q}}^{T} \mathbf{d}\right\} \text { is maximum, }
$$

under the following linear constraints:

$$
\begin{aligned}
& \mathbf{A d} \leq \mathbf{0} \\
& \mathbf{B d}=\mathbf{0} \\
& \mathbf{C d}=\hat{\mathbf{u}}_{0},
\end{aligned}
$$

where $\mathbf{A}, \mathbf{B} \in \mathbb{R}^{N_{c o l, p} \times 3 N_{b}}, \mathbf{C} \in \mathbb{R}^{N_{c o l, ~} \times 3 N_{b}}$ and $\hat{\mathbf{u}}_{0}$ is a vector whose elements are the values of the function $\mathbf{u}_{0}(\boldsymbol{x})$, representing the non-homogeneous essential boundary conditions, evaluated at collocation points on $\Gamma$. Constraints (63) and (64) represent the requirement that $v_{n} \leq 0$ on $\Gamma_{c}$ and $v_{t}=0$ on $\Gamma_{c}$ respectively, $\Gamma_{c}$ being the union of all the possible contact curves among the blocks. On the other hand, constraint (65) represents the non-homogeneous essential boundary conditions (i.e. foundation settlements) on $\Gamma_{u}$. It should be noticed that the objective function in (62) is the work of the assigned external loads. Moreover, observe that constraints (64) and (65) can be formally condensed into a single equality constraint:

$$
\left[\begin{array}{l}
\mathbf{B} \\
\mathbf{C}
\end{array}\right] \mathbf{d}=\left[\begin{array}{l}
\mathbf{0} \\
\hat{\mathbf{u}}_{0}
\end{array}\right]
$$

Finally, let us now consider the boundary minimization problem (57), assuming identically zero initial gap $\Delta(\boldsymbol{x})$ along $\Gamma_{c}$ : 


$$
(p, r)=\arg \inf _{\lambda \in L^{*}, \rho \in R}\left(\Pi_{\Delta}^{* r}(\rho)\right)=\arg \inf _{\lambda \in L^{*}, \rho \in R}\left(\sum_{i=1}^{N_{b}} c_{u, i}(\rho)\right)
$$

After discretization, the Lagrangean functional $\Pi_{\Delta}^{* r}$ associated to a given vector of boundary tractions $\mathbf{r} \in \mathbb{R}^{N_{c o l, r}}$ on $\Gamma_{u}$ can be expressed as a linear function of $\mathbf{r}$ as follows:

$$
\Pi_{\Delta}^{* r}(\mathbf{r})=\hat{\mathbf{u}}_{0}{ }^{T} \mathbf{r}
$$

Moreover, let $\left(\mathbf{p}_{n}, \mathbf{p}_{t}\right) \in \mathbb{R}^{2 N_{c o l, c}}$ be the discrete vector of normal and tangential components of normal contact tractions on $\Gamma_{c}$ respectively. Then, the discrete LP problem associated to (67) reads:

$$
\text { find }\left(\mathbf{p}_{n}, \mathbf{p}_{t}, \mathbf{r}\right) \text { s.t. }\left\{\hat{\mathbf{u}}_{0}{ }^{T} \mathbf{r}\right\} \text { is minimum, }
$$

under the following linear constraints:

$$
\begin{gathered}
\mathbf{p}_{n} \geq \mathbf{0} \\
\boldsymbol{A}^{T} \mathbf{p}_{n}+\mathbf{B}^{T} \mathbf{p}_{t}+\mathbf{C}^{T} \mathbf{r}-\hat{\mathbf{q}}=\mathbf{0},
\end{gathered}
$$

where constraint (70) represents the requirement that $p_{n} \geq 0$, and constraints (71) represents three equilibrium equations for each block (two translations and one rotations), see Remark 2. Here, the objective function is the work of constraint reactions for the settlements $\hat{\mathbf{u}}_{0}$. It is easy to verify that the LP problem (69),(70),(71) is the dual of the LP problem (62),(63),(66).

Remark 3. It is worth to compare the proposed formulations to the ones classically adopted in the framework of limit analysis of masonry structures and to underline the deep differences among them. As an example, in the kinematic theorem of limit analysis the variables are the rates of displacements induced by the failure mechanism, and the power of external dead loads (and internal dissipation) is minimized, under the constraint of the so-called normalization condition, which imposes that the power of live loads is equal to one. Furthermore, in the context of standard limit analysis it is not possible, as it is well known, to take into account the effects of assigned constraint settlements on the magnitude of the collapse load (Como, 2013). On the contrary, the proposed primal formulation, which rigorously accounts for the presence of settlements, simply provides that the work of external dead loads is maximized. In addition, the static theorem of limit analysis maximizes the multiplier of the live loads under equilibrium and admissibility constraints, while the proposed complementary formulation searches for the minimum, under the same constraints, of the work of external constraint reactions for the assigned settlements. 


\section{Examples}

As previously discussed, the LP formulations presented in the previous Section are formally very similar to the ones used in the limit analysis of masonry structures under the framework of Heyman's model (Chiozzi et al., 2017, 2016; Gilbert et al., 2006). The aim of this Section is to clearly illustrate, by means of numerical examples, how the proposed formulations allow not only to evaluate the effects of settlements but also to provide a sound foundation to the correct estimation of the load safety factor in masonry structures where settlements took place.

The first example is a simply supported masonry lintel composed by a single layer of masonry bricks, with a span of $1.5 \mathrm{~m}$, thickness of $0.24 \mathrm{~m}$, shown in Fig. 6(a). A vertical load equal to $1 \mathrm{kN} / \mathrm{m}$ has been applied to the extrados. A given horizontal displacement is applied to the second extremity of the lintel. The proposed contact formulation (62) straightforwardly allows to compute the deformed configuration, as shown in Fig. 6(b). Let us point out here that, for small to moderate horizontal settlements, unless masonry finite compression is taken into account, the structure at hand is "locked" and failure cannot be attained for any value of the applied external load. This basic result is wellknown and was illustrated by Heyman in his studies on the stability of masonry arches (Heyman, 1969). On the other hand, if masonry finite compression strength is accounted for, the limit load multiplier depends on the assumed horizontal displacement. Applying classic limit analysis to the deformed geometry computed by means of the proposed contact LP formulation (62), we find that the load multiplier decreases, as depicted in Fig. 7, and vanishes when the horizontal assigned displacement is equal to half of the thickness.

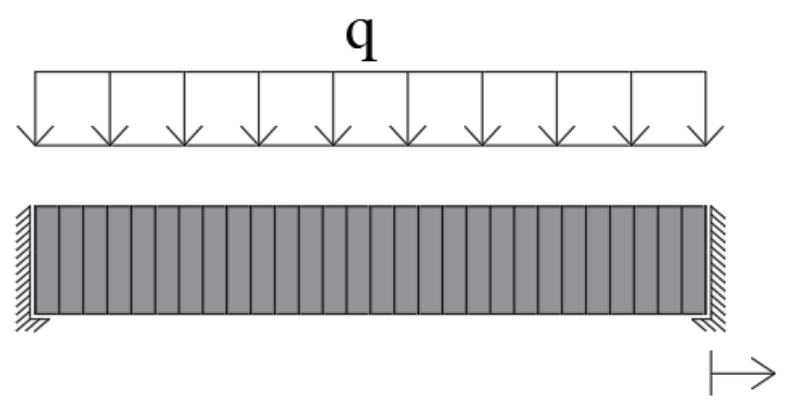

(a)

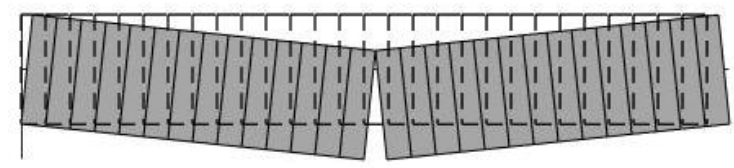

(b)

Fig. 6: Masonry lintel: (a) load condition and constraints, (b) deformed configuration due to a horizontal settlement of $2 \mathrm{~cm}$. 


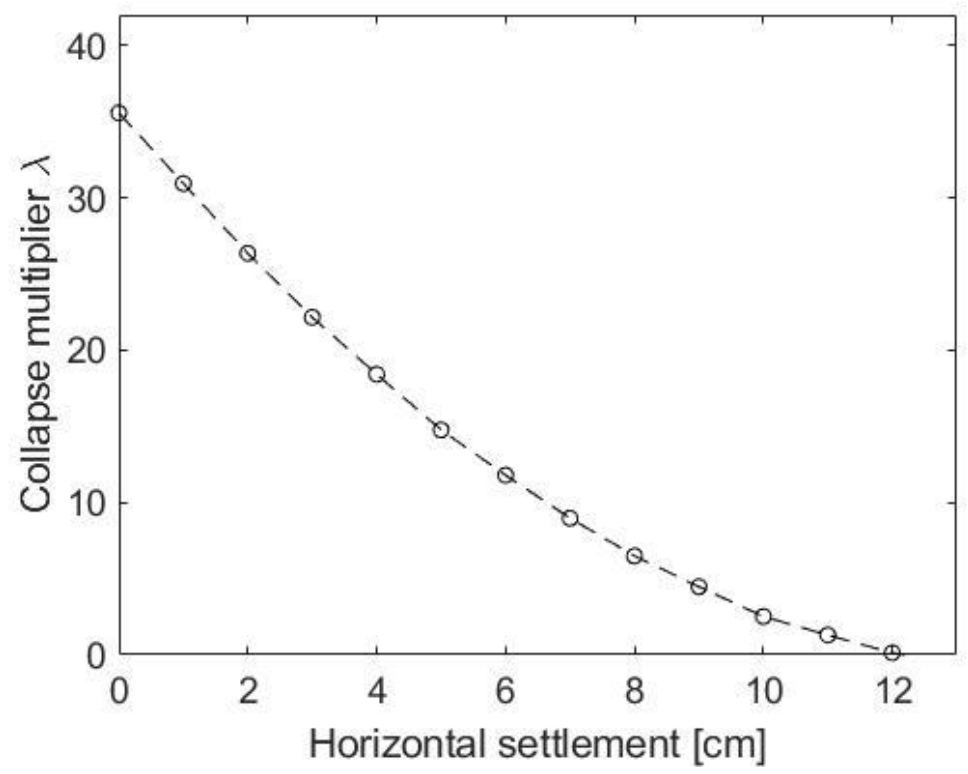

Fig. 7: Dependence of the collapse load multiplier on the assigned horizontal settlement, from limit analysis (including finite masonry compression strength).

For these computations a masonry compression strength of $2.4 \mathrm{MPa}$ has been assumed.

The second example we are presenting is a masonry semicircular arch. Again, the aim is not only to compute the deformed configuration due to a given settlement but also the variation in the load bearing capacity, associated with a certain variable load, because of that settlement. The load bearing capacity of the initial structure is evaluated by adopting a traditional limit analysis procedure, whereas the proposed contact LP formulation has been applied to obtain the deformed configuration following the application of the settlement.

As depicted in Fig. 8(a), the arch presents a radius of $2.86 \mathrm{~m}$, a thickness of $0.33 \mathrm{~m}$ and unitary width. Moreover, 64 masonry bricks compose the arch. The dead loads are due to self-weight, where a specific weight equal to $18 \mathrm{kN} / \mathrm{m}^{3}$ is assumed for masonry. As a variable load, a uniform vertical pressure $\mathrm{q}=1 \mathrm{kN} / \mathrm{m}^{2}$ has been applied. The collapse load multiplier obtained through standard limit analysis for the structure without settlements is equal to 0.429, see Fig. 8(b).

The first applied settlement is a horizontal displacement of the first abutment. The corresponding deformed configuration, computed through the proposed contact LP formulation (62), is shown in Fig. 9. As a consequence of the horizontal settlement, three symmetrical hinges can be observed on the arch: one at the arch crown and the others up to the thirteenth voussoir. After the application of the settlement, the load bearing capacity has been re-evaluated through limit analysis. As already observed in the previous section, it must be stressed here that the load bearing capacity can by determined by means of standard limit analysis only if the collapse mechanism does not entail the closure of some existing open hinges due to the settlement. 


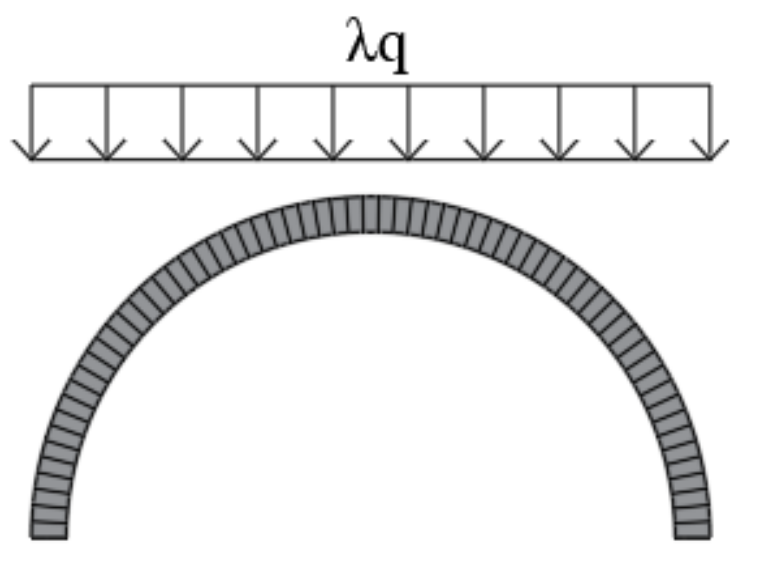

(a)

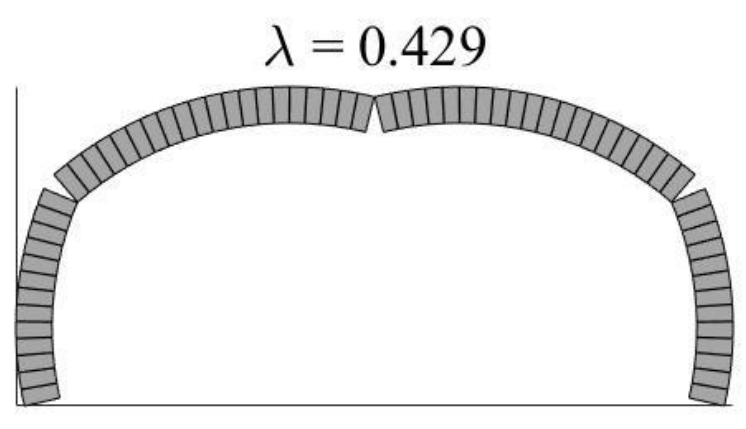

(b)

Fig. 8: Semicircular arch: (a) uniform vertical load, (b) load bearing capacity and collapse mechanism.

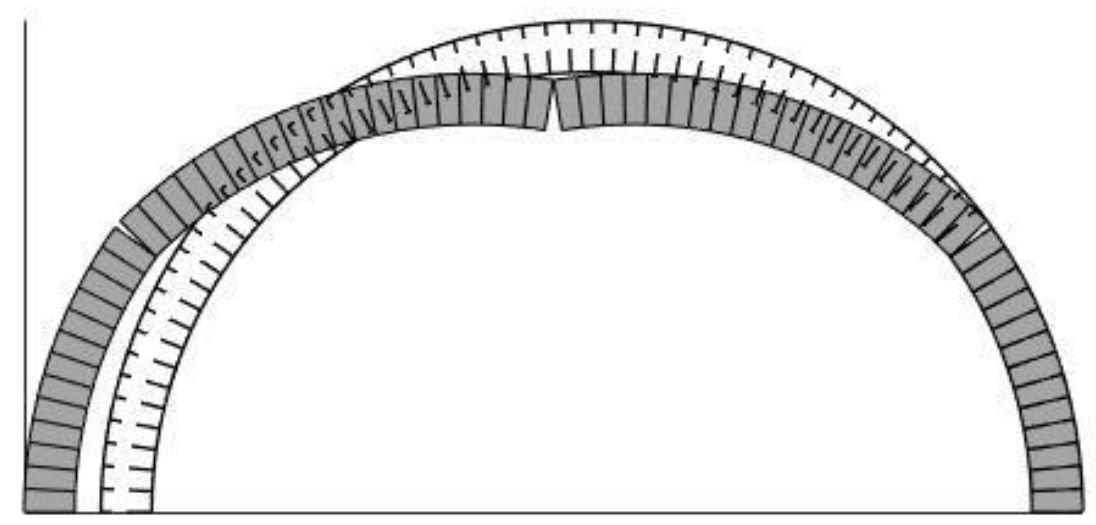

Fig. 9: Deformed configuration due to a $50 \mathrm{~cm}$ horizontal settlement of one pier.

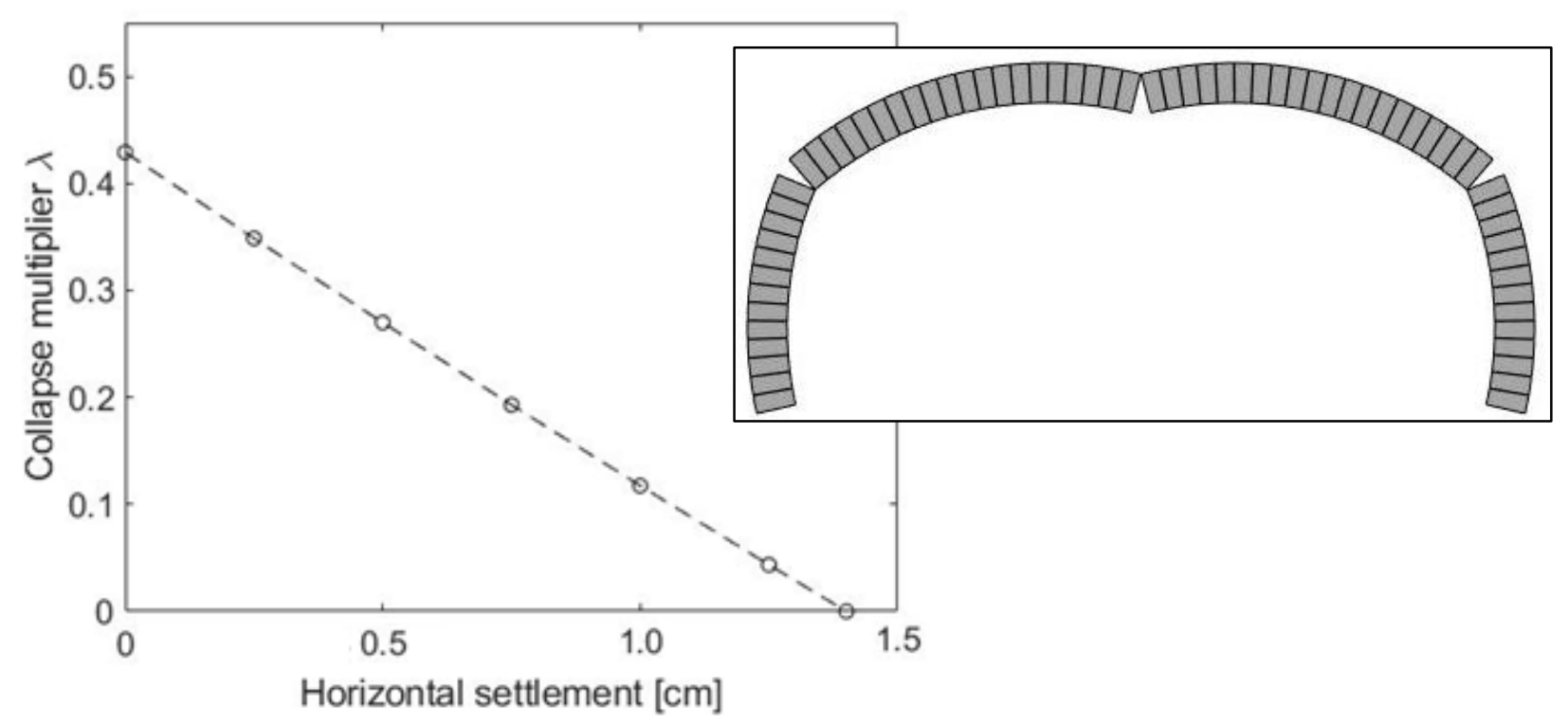

Fig. 10: Dependence of the collapse load multiplier on the assigned horizontal settlement. 
In the problem at hand, since the hinges due to the settlements are the same that are produced by the collapse mechanism due to vertical loads shown in Fig. 8(b), the new load bearing capacity can be obtained by applying the standard limit analysis procedure to the deformed configuration provoked by the initial settlement. As a result, a lower load multiplier is found. In Fig. 10 the collapse multipliers obtained for different values of horizontal settlements are depicted together with the collapse mechanism of the deformed structure. Notably, the load bearing capacity decreases linearly with the settlement (linearity is due to the high slenderness of the arch) and for a horizontal settlement equal to $1.4 \mathrm{~cm}$ the equilibrium of the arch is no more verified under any positive vertical load. As expected, in this case the collapse mechanism is qualitatively the same of the perfect structure, i.e. the structure without applied constrain settlements.

Let us now consider the case of a vertical settlement applied to the first abutment. Fig. 11 shows the deformed configuration, computed with the proposed contact LP formulation (62). In this case, there is no symmetry in the deformed structure. No hinges appear on the first half of the arch, which moves vertically, whereas three hinges appear to the other half, one at the arch crown, one at the base and the last up to the thirteenth voussoir from the right.

As in the previous case, the load bearing capacity under the vertical load distribution after the settlement can be evaluated by means of standard limit analysis because the collapse mechanism obtained for the deformed structure (depicted in Fig. 11) takes place by opening new hinges and emphasizing the hinges which are already open due to the settlement. No closure of existing hinges is entailed by the collapse mechanism. The load bearing capacity evaluated for different values of vertical settlements is shown in Fig. 12, together with the collapse mechanism. It can be noted that the collapse multiplier assumes a null value for very small values of the vertical settlement: for a vertical settlement of $0.42 \mathrm{~cm}$ the equilibrium under dead loads is not possible anymore.

In the examples discussed so far, the load bearing capacity of the deformed structure can be evaluated in an easy way because the collapse mechanism evolves accordingly to the already open hinges produced by applied settlements. However, this situation does not occur for every possible load configuration. For some load configurations, the collapse mechanism of a structure deformed by settlements entails the closure of some of the existing open hinges; namely, different inequality constraints describing the contact problem are required whenever a hinge is closed. In other words, during unloading masonry behaves as a nonlinear elastic material and not as a plastic material. In this situation, in which the behavior at each interface changes as the mechanism evolves, standard limit analysis is not applicable. Therefore, in the evaluation of the load bearing capacity, an incremental procedure in which the collapse mechanism is checked and updated at each step is needed. 


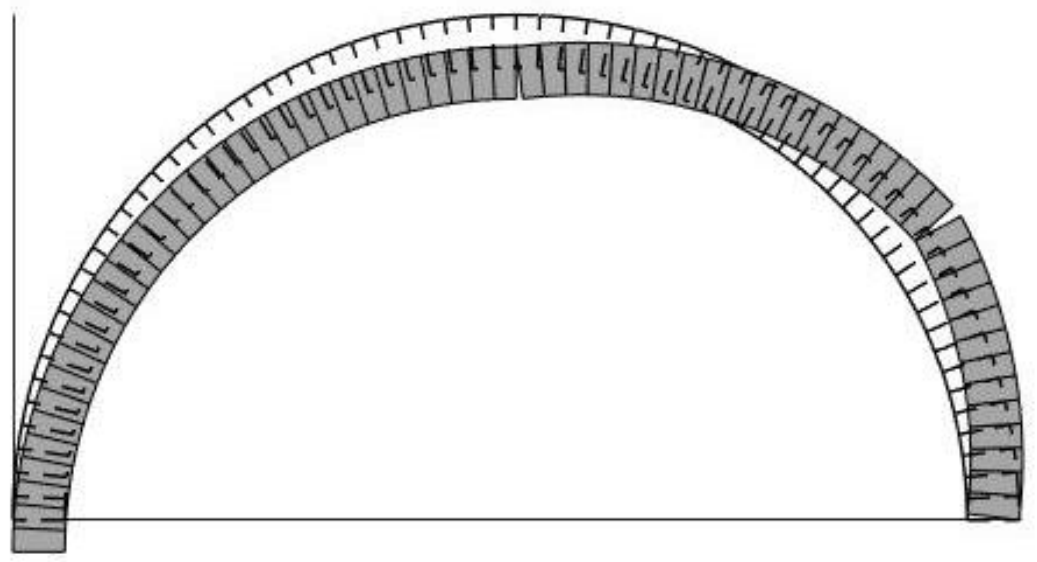

Fig. 11: Displacement configuration due a $20 \mathrm{~cm}$ vertical settlement of one pier.

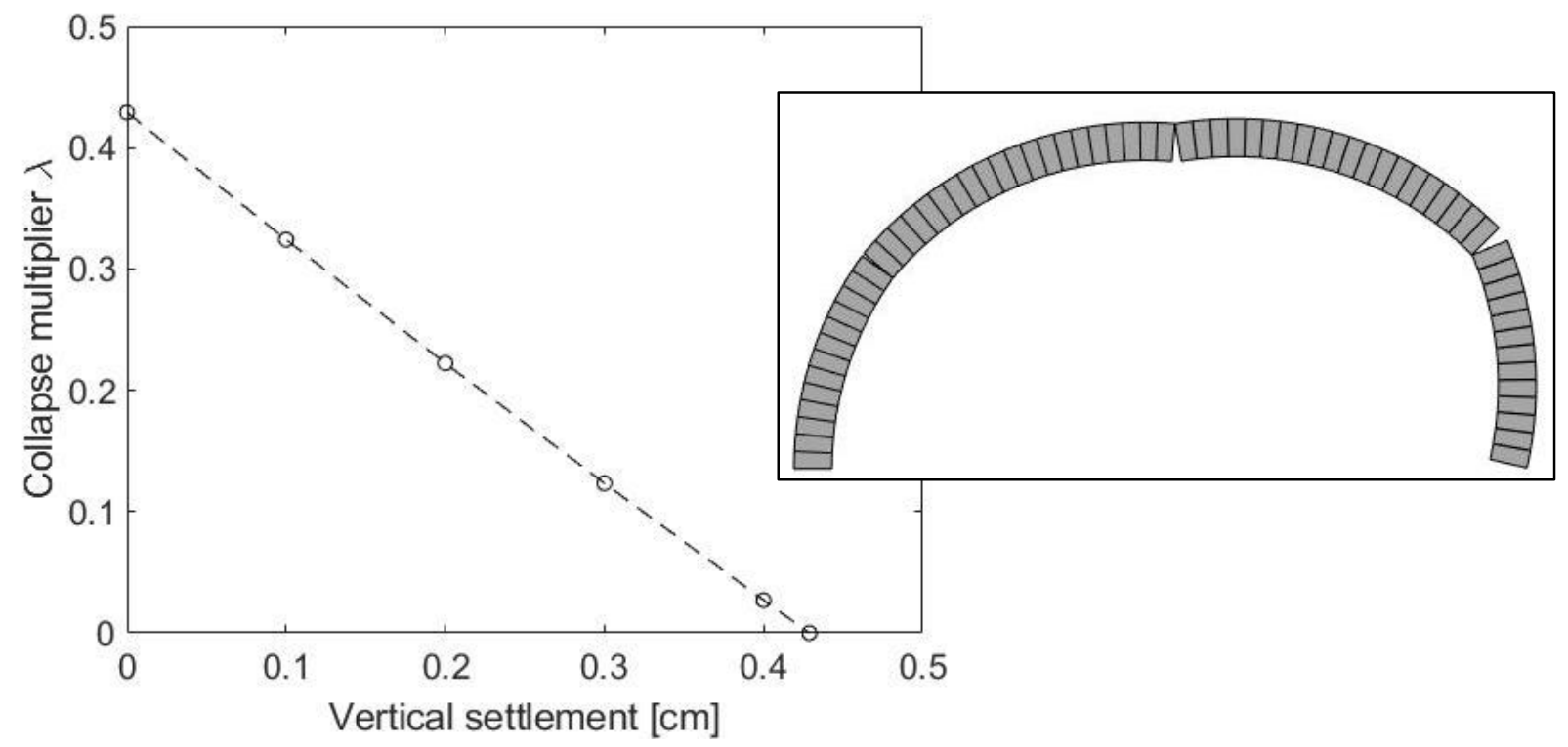

Fig. 12: Dependence of the collapse load multiplier on the assigned vertical settlement.

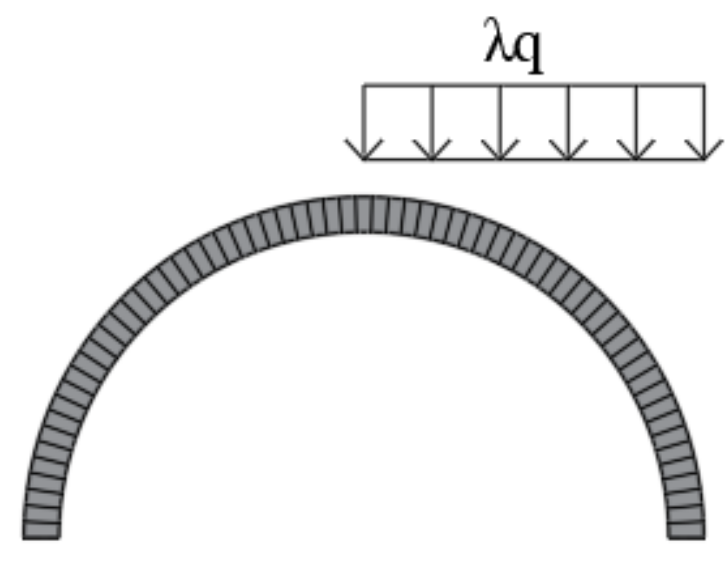

(a)

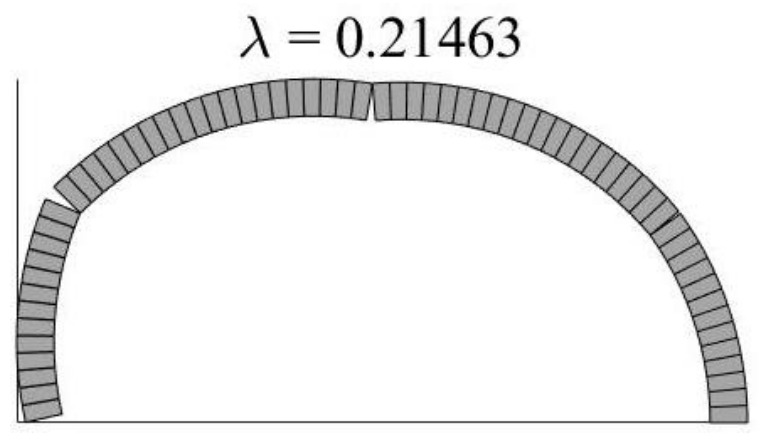

(b)

Fig. 13: Semicircular arch: (a) vertical load on a half of the span, (b) load bearing capacity and collapse mechanism. 
Let us consider the same masonry arch previously analyzed, vertically loaded on a half of the span, as depicted in Fig. 13(a). The collapse load multiplier, of the arch in absence of settlements is equal to 0.215: the corresponding collapse mechanism is depicted in Fig. 13(b). We apply the same vertical settlement at the first abutment considered in the previous example. The deformed configuration, computed using (62), is the same shown in Fig. 11. Note that the vertical settlement is applied on the opposite pier relatively to the position of the vertical load. Taking into account the deformed structure depicted in Fig. 11, we observe that the collapse mechanism due to the considered load, represented in Fig. 13(b), does not occur simply by emphasizing the opening of existing hinges formed because of the settlement. For this reason, an incremental analysis of the arch appears necessary.

At step one, we apply standard limit analysis to determine the candidate collapse mechanism from the computed deformed configuration. Then, in a number of sub-steps, we use the principle of virtual works to compute the collapse load multiplier as the mechanism evolves, performing, in fact, a nonlinear kinematic analysis. The evolution of the mechanism is controlled by the displacement of a suitable control point. If, at the current sub-step, one or more open hinges are closing, the inequality constraints, describing the new interface contact conditions, are updated in the limit analysis LP formulation: therefore, a different candidate collapse mechanism is found and the dependence of the collapse load multiplier on the evolution of the new mechanism is evaluated by means of the principle of virtual works in the subsequent sub-steps.

Fig. 14 shows the result of the application of the nonlinear kinematic analysis to the undeformed arch. The control point is at the arch crown and its horizontal displacement $d x$ has been chosen as the parameter describing the evolution of the mechanism (see Fig. 14(a)).

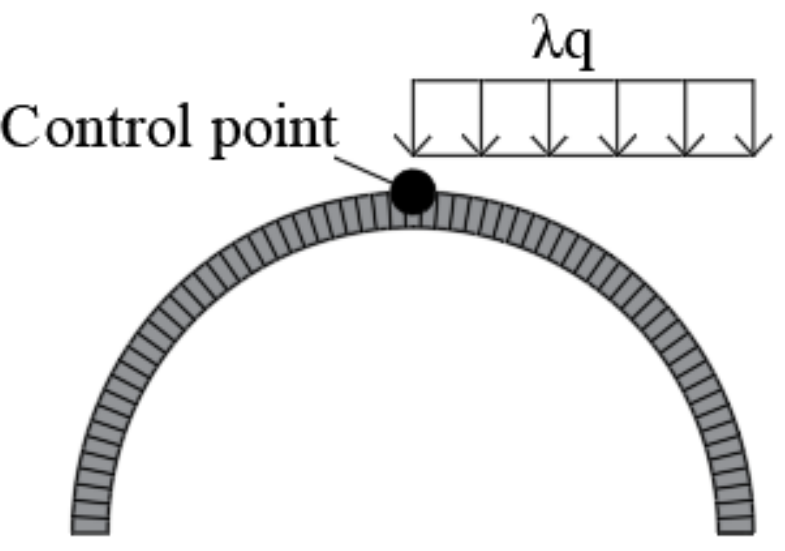

(a)

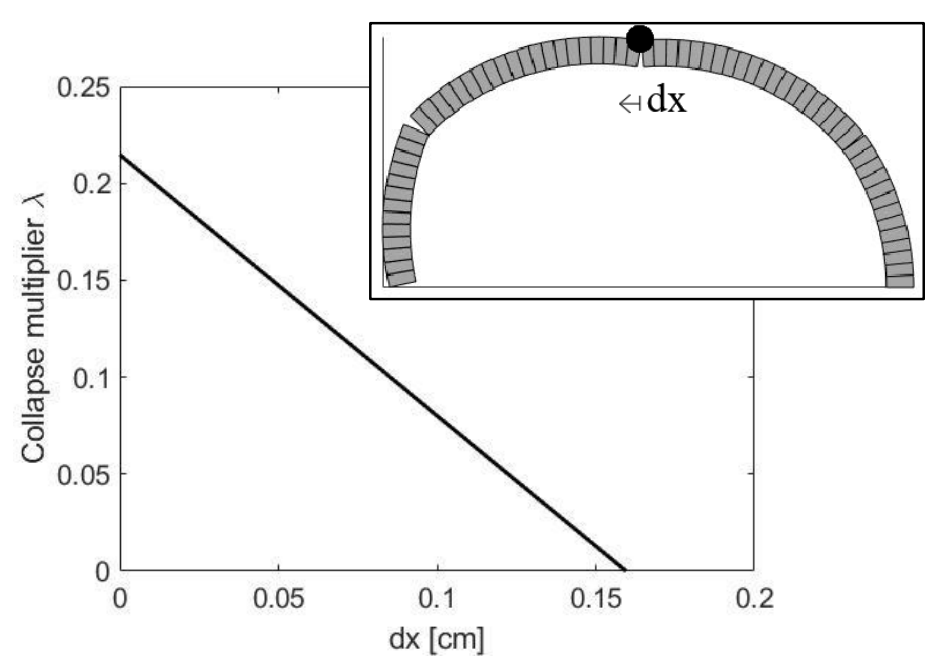

(b)

Fig. 14: Incremental analysis of the perfect structure: (a) control point adopted, (b) diagram $\lambda$-dx and corresponding collapse mechanism. 


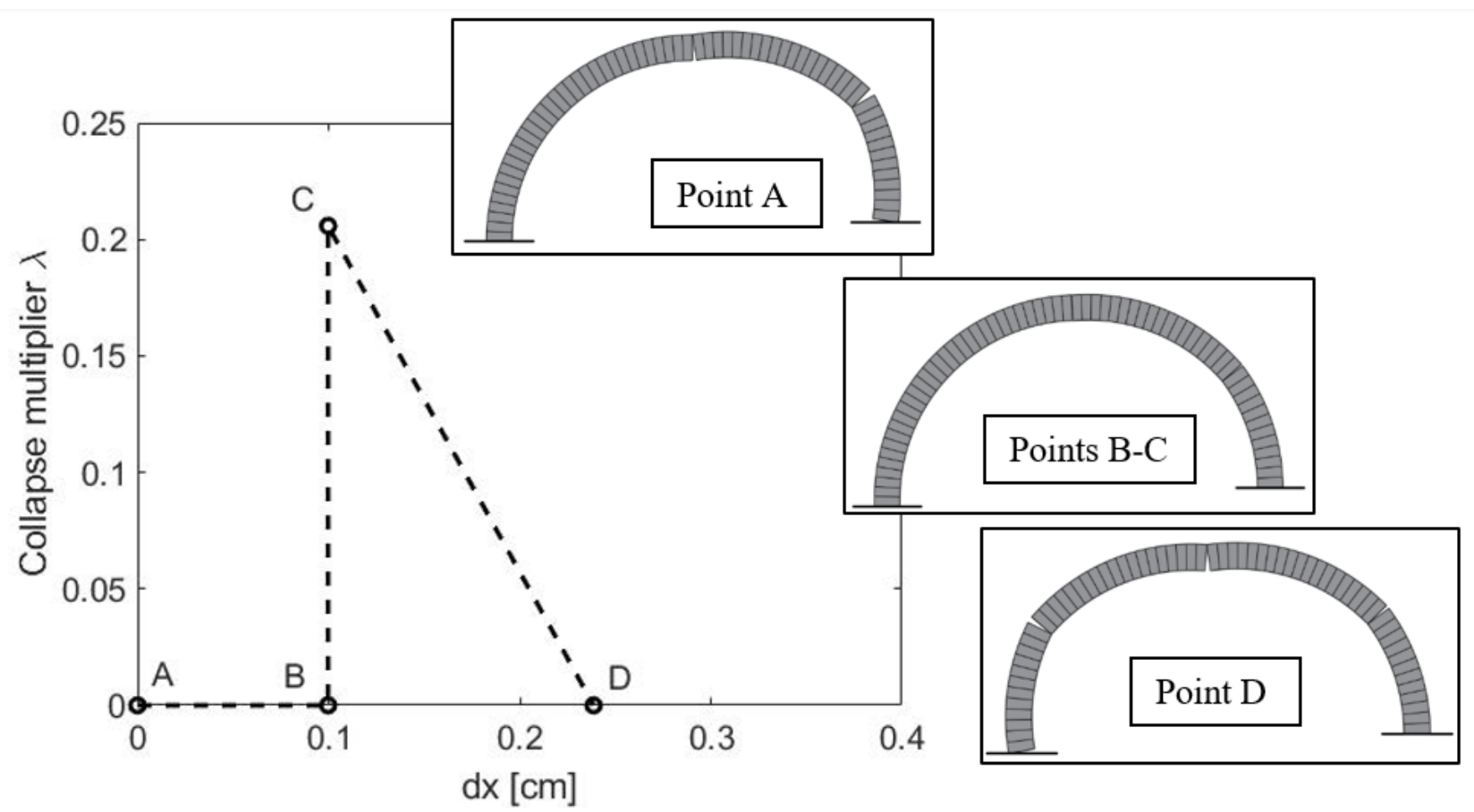

Fig. 15: Incremental analysis of the arch subjected to an initial vertical settlement at the first abutment equal to $0.25 \mathrm{~cm}$.

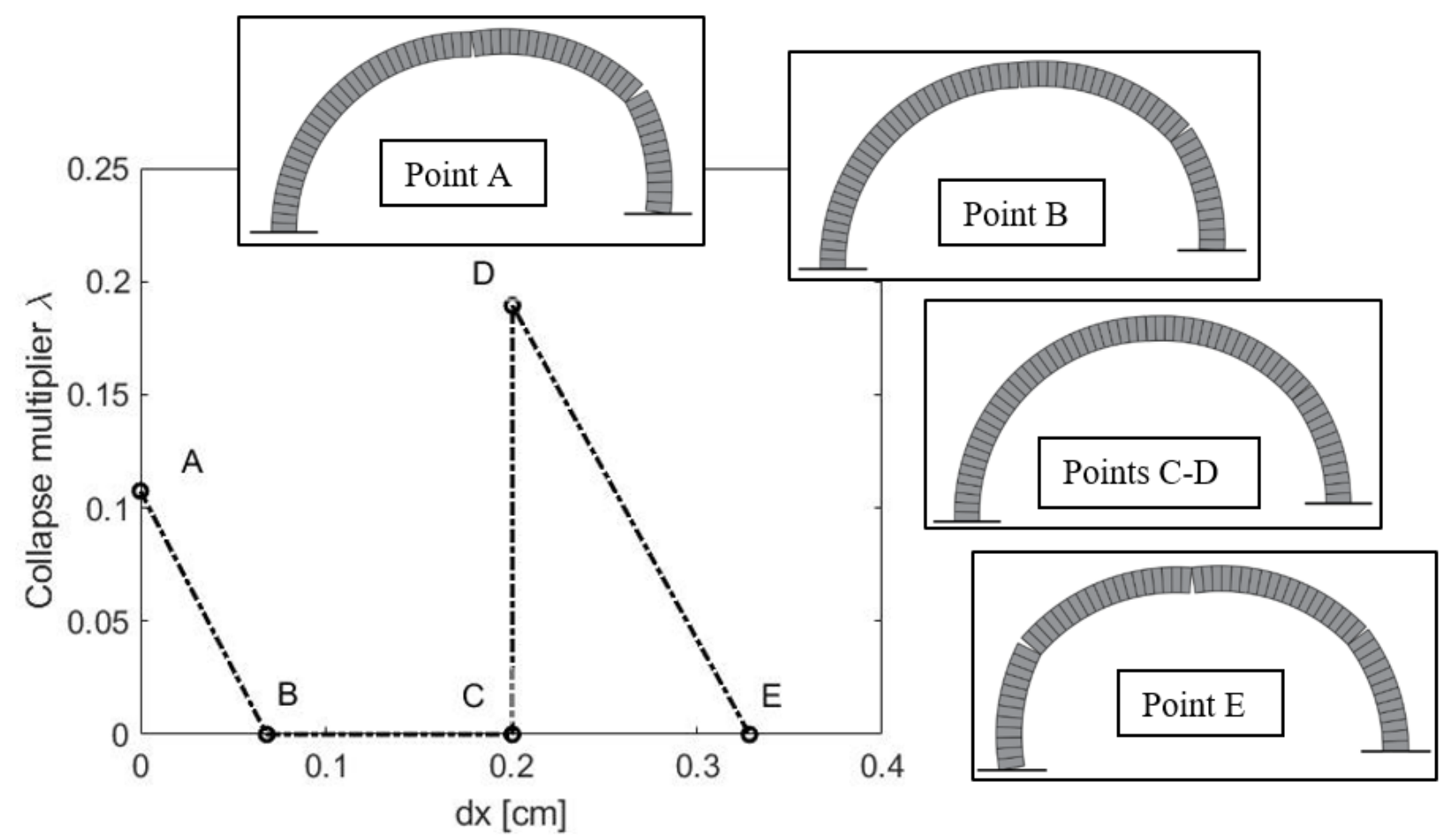

Fig. 16: Incremental analysis of the arch subjected to an initial vertical settlement at the first abutment equal to $0.5 \mathrm{~cm}$.

Due to the slenderness of the arch, the load multiplier decreases linearly with the evolution of the mechanism. 


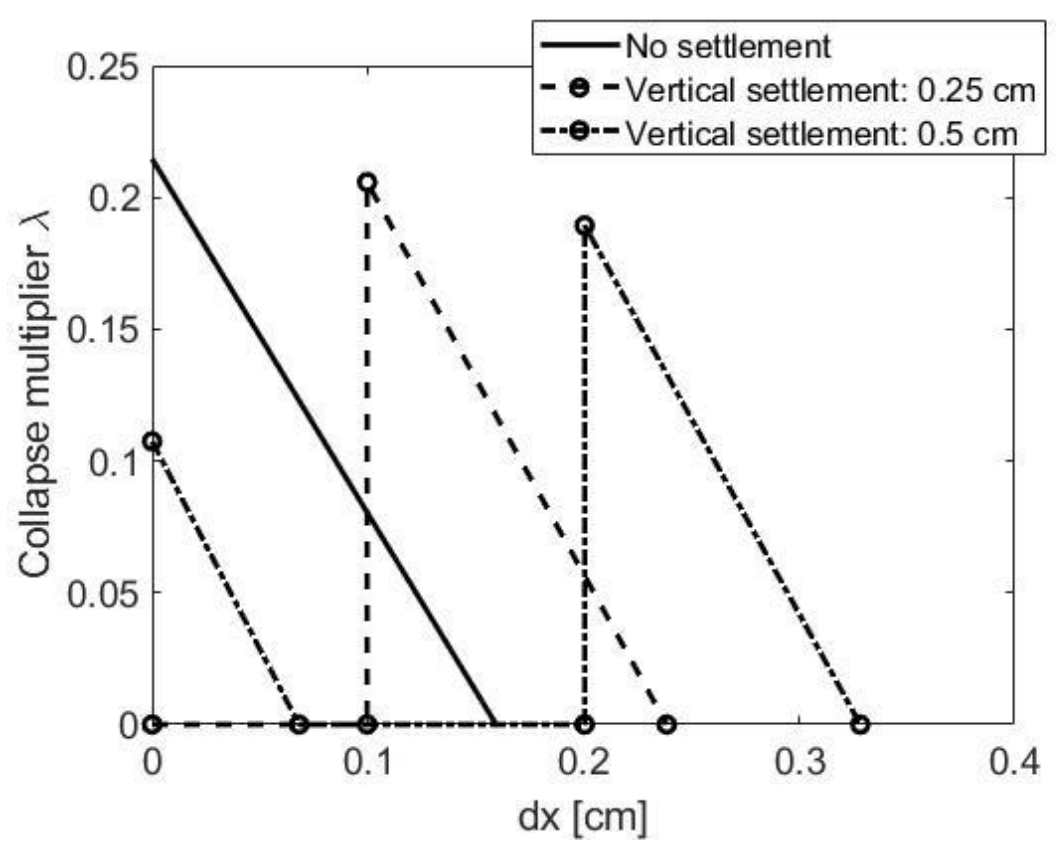

Fig. 17: Comparison between the obtained force-displacement diagrams.

When the incremental procedure is applied to an initially deformed structure, such as a structure undergoing settlements, the load decreases linearly but discontinuities in the force-displacement response are observed whenever an open hinge closes. The procedure has been applied to the arch subjected to two different values of vertical settlement of the first abutment.

The first applied vertical settlement is of magnitude $0.25 \mathrm{~cm}$ and the obtained force-displacement response is shown in Fig. 15. In the range A-B the initially open hinges start closing under the action of self-weight, so in the evolution of the mechanism a null value of load multiplier is obtained. At point $\mathrm{B}$ the hinge at the base is completely closed, and the structure can now sustain a non-null value of the applied load, therefore a jump in the load multiplier appears. After point $\mathrm{C}$, no other hinges close, and the load multiplier decreases linearly until the collapse.

The second value of vertical settlements considered is $0.5 \mathrm{~cm}$. Fig. 16 shows the obtained forcedisplacement response. In this case, the self-weight it is not sufficient to close the hinge at the base and a non-null value of vertical load is needed to start the evolution of the mechanism: so, differently to the previous case, point $\mathrm{A}$ does not coincide with the origin. At point $\mathrm{B}$, the same collapse mechanism evolves under the action of self-weight only. At point $\mathrm{C}$, the hinge at the base is completely closed, the mechanism evolves differently and a higher load multiplier is needed. Analogously to the previous case, after point D the load multiplier decreases linearly until zero.

Finally, all the obtained force-displacement diagrams are summarized in Fig. 17. It can be observed that the maximum value of the load multiplier decreases for higher vertical settlements. However, these values can be obtained only through an incremental analysis of the collapse mechanism by 
means of the proposed contact linear programming procedure and not by standard limit analysis. In fact, given the unilateral behavior assigned to masonry interfaces, since stress at each interface is not monotonic during the evolution of the mechanism, standard limit analysis cannot be applied.

Obviously, aside from the considerations regarding the assessment of the load bearing capacity described in the previous paragraphs, the proposed formulations allow for the computation of the fractured configuration of general masonry structures undergoing settlements. As an example, we report the case of the façade of a historical masonry church in Bondeno (Italy), with a uniform applied settlement of $20 \mathrm{~cm}$ along half of its length. The façade is 19 meter high and is built with a typical Romanesque style with three rose windows and three pointed arch doors, see (Chiozzi et al., 2018) for a detailed description of the façade geometry.

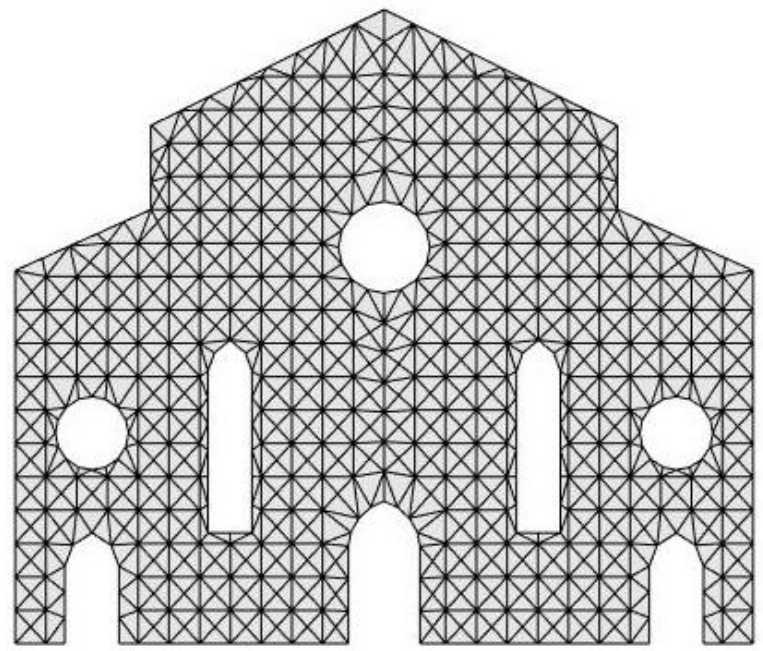

(a)

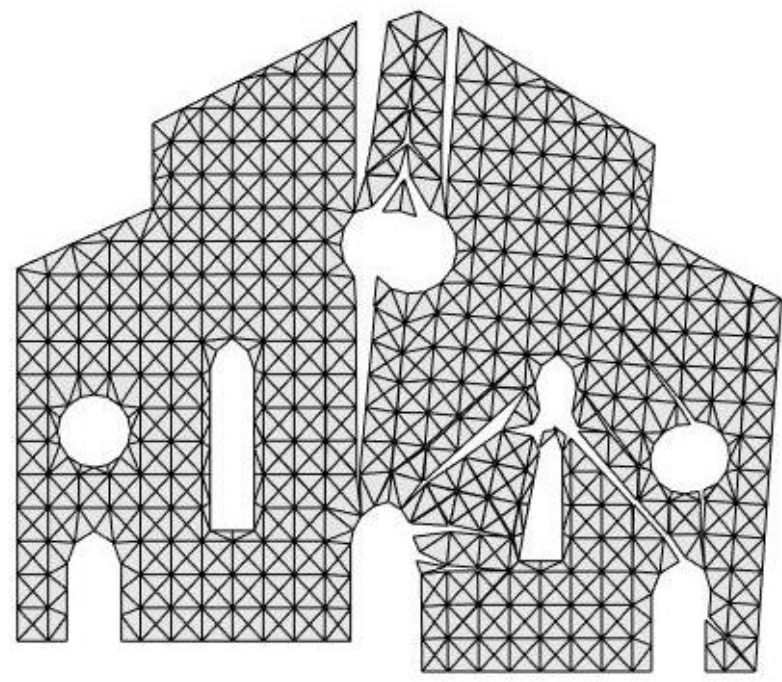

(b)

Fig. 18: Masonry church façade: (a) undeformed configuration mesh; (b) scaled plot of the fractured configuration after the application of the settlements.

Fig. 18(a) represents a structured mesh of rigid elements chosen for the façade, while Fig. 18(b) represents a plot of the fractured configuration after the settlement is applied, computed by means of the contact LP formulation (62) and scaled for the sake of clarity. It is worth pointing out that, unless the mesh is purposely constructed to represent the actual arrangement of masonry bricks, the use of plain LP formulations such as (62) on three-dimensional masonry elements may lead to a certain grade of mesh-dependency in the solution, which can be overcome by means of suitable optimization algorithms. Such extension of the proposed approach is out of the scope of the present paper and will be the subject of an upcoming work by the Authors. 


\section{Conclusions}

In this paper we propose a new perspective on the modeling of the influence of foundation settlements in masonry structures. For a given masonry structure, we show that it is possible to model in a consistent way the crack pattern and the associated mechanism induced by applied settlements starting from a proper treatment of unilateral contact constraints arising between contiguous blocks, in which the structure is partitioned after settlements have taken place. In particular, we derive two variational formulations and their discretization directly entails two dual linear programming problems. The first one, which classically derives from the minimization of total potential energy, maximizes the work of external loads, whilst the second one, which appears new in the literature, minimizes the work of constraint reactions for the assigned displacements

Finally, we present simple but technically relevant examples useful to illustrate the peculiarities of the proposed approach. We show how the proposed formulations can not only evaluate the effect of settlements but also how the problem of assessing the load safety factor in masonry structures in the presence of settlements can be dealt within the framework of classical Heyman's hypotheses on the behavior of masonry material.

\section{References}

Alliney, S., Tralli, A., Alessandri, C., 1990. Boundary variational formulations and numerical solution techniques for unilateral contact problems. Comput. Mech. 6, 247-257. https://doi.org/10.1007/BF00370105

Ambrosio, L., De Giorgi, E., 1988. A new kind of functional in the calculus of variations (in italian). Atti della Accad. Naz. dei Lincei, Rend. della Cl. di Sci. Fis. Mat. e Nat. 8, 199-210.

Angelillo, M., 2014. Practical applications of unilateral models to Masonry Equilibrium, in:

Mechanics of Masonry Structures. Springer, Vienna, pp. 109-210. https://doi.org/10.1007/9783-7091-1774-3_4

Angelillo, M., 1993. Constitutive relations for no-tension materials. Meccanica 28, 195-202. https://doi.org/10.1007/BF00989121

Angelillo, M., Fortunato, A., Gesualdo, A., Iannuzzo, A., Zuccaro, G., 2018. Rigid block models for masonry structures. Int. J. Mason. Res. Innov. 3, 349. https://doi.org/10.1504/IJMRI.2018.095701

Angelillo, M., Fortunato, A., Montanino, A., Lippiello, M., 2014. Singular stress fields in masonry structures: Derand was right. Meccanica 49, 1243-1262. https://doi.org/10.1007/s11012-014- 
9880-6

Antes, H., Panagiotopoulos, P.D., 1992. The Boundary Integral Approach to Static and Dynamic Contact Problems. Equalitiy and Inequality Methods. Birkhäuser Verlag.

Boothby, T.E., 2001. Analysis of masonry arches and vaults. Prog. Struct. Eng. Mater. 3, 246-256. https://doi.org/10.1002/pse.84

Chambolle, A., Giacomini, A., Ponsiglione, M., 2007. Piecewise rigidity. J. Funct. Anal. 244, 134153. https://doi.org/10.1016/J.JFA.2006.11.006

Chiozzi, A., Grillanda, N., Milani, G., Tralli, A., 2018. UB-ALMANAC: An adaptive limit analysis NURBS-based program for the automatic assessment of partial failure mechanisms in masonry churches. Eng. Fail. Anal. 85, 201-220. https://doi.org/10.1016/j.engfailanal.2017.11.013

Chiozzi, A., Malagù, M., Tralli, A., Cazzani, A., 2016. ArchNURBS: NURBS-Based Tool for the Structural Safety Assessment of Masonry Arches in MATLAB. J. Comput. Civ. Eng. 30. https://doi.org/10.1061/(ASCE)CP.1943-5487.0000481

Chiozzi, A., Milani, G., Tralli, A., 2017. A Genetic Algorithm NURBS-based new approach for fast kinematic limit analysis of masonry vaults. Comput. Struct. 182, 187-204. https://doi.org/10.1016/j.compstruc.2016.11.003

Coccia, S., Di Carlo, F., Rinaldi, Z., 2015. Collapse displacements for a mechanism of spreadinginduced supports in a masonry arch. Int. J. Adv. Struct. Eng. 7, 307-320. https://doi.org/10.1007/s40091-015-0101-x

Como, M., 2017. Virtual displacements principle, existence and uniqueness for elastic no tension bodies. Meccanica 52, 1397-1405. https://doi.org/10.1007/s11012-016-0480-5

Como, M., 2013. Statics of Historic Masonry Constructions. Springe-Verlag Berlin Heidelberg. https://doi.org/10.1007/978-3-642-30132-2

Como, M., 1992. Equilibrium and collapse analysis of masonry bodies. Meccanica 27, 185-194. https://doi.org/10.1007/BF00430044

Dalgic, K.D., Hendriks, M.A.N., Ilki, A., Broere, W., 2018. A two-stage numerical analysis approach for the assessment of the settlement response of the pre-damaged historic Hoca Pasha Mosque. Int. J. Archit. Herit. 1-21. https://doi.org/10.1080/15583058.2018.1469174

Del Piero, G., 1998. Limit analysis and no-tension materials. Int. J. Plast. 14, 259-271. https://doi.org/10.1016/S0749-6419(97)00055-7

Di Carlo, F., Coccia, S., Rinaldi, Z., 2018. Collapse load of a masonry arch after actual displacements of the supports. Arch. Appl. Mech. 88, 1545-1558. https://doi.org/10.1007/s00419-018-1386-6

Drucker, D.C., 1959. A definition of stable inelastic material. J. Appl. Mech. 26, 101-106. 
Facchinei, F., Pang, J.-S. (Eds.), 2004. Finite-Dimensional Variational Inequalities and Complementarity Problems, Springer Series in Operations Research and Financial Engineering. Springer New York, New York, NY. https://doi.org/10.1007/b97543

Fichera, G., 1972. Boundary value problems in elasticity with unilateral constraints. Encycl. Phys. (Edited by S. Flügge).

Fichera, G., 1964. Elastostatic problems with unilateral constraints: the Signorini problem with ambigous boundary conditions (in italian). Rend. della Accad. Naz. dei Lincei, Cl. di Sci. Fis. Mat. e Nat. 8 34, 138-142.

Galassi, S., Misseri, G., Rovero, L., Tempesta, G., 2018. Failure modes prediction of masonry voussoir arches on moving supports. Eng. Struct. 173, 706-717. https://doi.org/10.1016/J.ENGSTRUCT.2018.07.015

Galassi, S., Paradiso, M., Tempesta, G., 2013. Non-Linear Analysis of Masonry Structures Subjected to External Settlements. Open J. Civ. Eng. 03, 18-26. https://doi.org/10.4236/ojce.2013.32A003

Galassi, S., Tempesta, G., 2019. The Matlab code of the method based on the Full Range Factor for assessing the safety of masonry arches. MethodsX 6, 1521-1542. https://doi.org/10.1016/J.MEX.2019.05.033

Gilbert, M., Casapulla, C., Ahmed, H.M., 2006. Limit analysis of masonry block structures with non-associative frictional joints using linear programming. Comput. Struct. 84, 873-887. https://doi.org/10.1016/j.compstruc.2006.02.005

Grioli, G., 1983. On the stress in rigid bodies. Meccanica 18, 3-7. https://doi.org/10.1007/BF02156094

Heyman, J., 1969. The safety of masonry arches. Int. J. Mech. Sci. 11, 363-385. https://doi.org/10.1016/0020-7403(69)90070-8

Heyman, J., 1966. The stone skeleton. Int. J. Solids Struct. 2, 249-279. https://doi.org/10.1016/0020-7683(66)90018-7

Huerta, S., 2011. The Analysis of Masonry Architecture: A Historical Approach. Archit. Sci. Rev. $51,297-328$.

Iannuzzo, A., Angelillo, M., De Chiara, E., De Guglielmo, F., De Serio, F., Ribera, F., Gesualdo, A., 2018. Modelling the cracks produced by settlements in masonry structures. Meccanica 53, 1857-1873. https://doi.org/10.1007/s11012-017-0721-2

Kikuchi, N., Oden, J.T., 1988. Contact problems in elasticity: a study of variational inequalities and finite element methods. SIAM.

Lucchesi, M., Šilhavý, M., Zani, N., 2006. A new class of equilibrated stress fields for no-tension 
bodies. J. Mech. Mater. Struct. 1, 503-539. https://doi.org/10.2140/jomms.2006.1.503

Panagiotopoulos, P.D., 1985. Inequality Problems in Mechanics and Applications. Convex and Nonconvex Energy Functions. Birkhäuser, Basel.

Portioli, F., Cascini, L., 2017. Large displacement analysis of dry-jointed masonry structures subjected to settlements using rigid block modelling. Eng. Struct. 148, 485-496. https://doi.org/10.1016/J.ENGSTRUCT.2017.06.073

Portioli, F., Cascini, L., 2016. Assessment of masonry structures subjected to foundation settlements using rigid block limit analysis. Eng. Struct. 113, 347-361. https://doi.org/10.1016/J.ENGSTRUCT.2016.02.002

Romano, A., Ochsendorf, J.A., 2010. The Mechanics of Gothic Masonry Arches. Int. J. Archit. Herit. 4, 59-82. https://doi.org/10.1080/15583050902914660

Šilhavý, M., 2014. Collapse mechanisms and the existence of equilibrium solutions for masonry bodies. Math. Mech. Solids 19, 821-831. https://doi.org/10.1177/1081286513488618

Tempesta, G., Galassi, S., 2019. Safety evaluation of masonry arches. A numerical procedure based on the thrust line closest to the geometrical axis. Int. J. Mech. Sci. 155, 206-221. https://doi.org/10.1016/j.ijmecsci.2019.02.036

Tralli, A., Alessandri, C., Milani, G., 2014. Computational Methods for Masonry Vaults: A Review of Recent Results. Open Civ. Eng. J. 8.

Wriggers, P., 2006. Computational Contact Mechanics. Springer Berlin Heidelberg, Berlin, Heidelberg. https://doi.org/10.1007/978-3-540-32609-0

Zampieri, P., Amoroso, M., Pellegrino, C., 2019. The masonry buttressed arch on spreading support. Structures 20, 226-236. https://doi.org/10.1016/J.ISTRUC.2019.03.008

Zampieri, P., Cavalagli, N., Gusella, V., Pellegrino, C., 2018a. Collapse displacements of masonry arch with geometrical uncertainties on spreading supports. Comput. Struct. 208, 118-129. https://doi.org/10.1016/J.COMPSTRUC.2018.07.001

Zampieri, P., Faleschini, F., Zanini, M.A., Simoncello, N., 2018b. Collapse mechanisms of masonry arches with settled springing. Eng. Struct. 156, 363-374. https://doi.org/10.1016/J.ENGSTRUCT.2017.11.048

Zampieri, P., Zanini, M.A., Faleschini, F., Hofer, L., Pellegrino, C., 2017. Failure analysis of masonry arch bridges subject to local pier scour. Eng. Fail. Anal. 79, 371-384. https://doi.org/10.1016/J.ENGFAILANAL.2017.05.028 